Published in final edited form as:

Nat Med. 2019 January ; 25(1): 82-88. doi:10.1038/s41591-018-0290-5.

\title{
Calibration of CAR activation potential directs alternative T cell fates and therapeutic potency
}

\author{
Judith Feucht ${ }^{1,4}$, Jie Sun ${ }^{1,2,4}$, Justin Eyquem ${ }^{1}$, Yu-Jui Ho ${ }^{3}$, Zeguo Zhao ${ }^{1}$, Josef Leibold ${ }^{3}$, \\ Anton Dobrin ${ }^{1}$, Annalisa Cabriolu ${ }^{1}$, Mohamad Hamieh ${ }^{1}$, and Michel Sadelain ${ }^{1,}{ }^{*}$ \\ ${ }^{1}$ Center for Cell Engineering and Immunology Program, Sloan Kettering Institute, Memorial Sloan \\ Kettering Cancer Center, New York, NY, USA. \\ ${ }^{2}$ Institute of Hematology, Zhejiang University \& Zhejiang Engineering Laboratory for Stem Cell \\ and Immunotherapy, Zhejing, China. \\ ${ }^{3}$ Cancer Biology and Genetics Program, Sloan Kettering Institute, Memorial Sloan Kettering \\ Cancer Center, New York, NY, USA. \\ ${ }^{4}$ These authors contributed equally: Judith Feucht, Jie Sun.
}

\begin{abstract}
Chimeric antigen receptors (CARs) are synthetic receptors that target and reprogram T cells to acquire augmented antitumor properties ${ }^{1}$. CD19-specific CARs that comprise CD28 and CD3 $\zeta$ signaling motifs ${ }^{2}$ have induced remarkable responses in patients with refractory leukemia ${ }^{3-5}$ and lymphoma ${ }^{6}$ and were recently approved by the US Food and Drug Administration ${ }^{7}$. These CARs program highly performing effector functions that mediate potent tumor elimination ${ }^{4,8}$ despite the limited persistence they confer on $\mathrm{T}$ cells ${ }^{3-6,8}$. Extending their functional persistence without compromising their potency should improve current CAR therapies. Strong T cell activation drives
\end{abstract}

\footnotetext{
Reprints and permissions information is available at www.nature.com/reprints.

*Correspondence and requests for materials should be addressed to M.S. m-sadelain@ski.mskcc.org. Author contributions

J.E. and Y.-J.H. share second authorship. J.F. and J.S. designed the study, performed the experiments, analyzed and interpreted the data, and wrote the manuscript. J.E. and A.D. performed CRISPR-Cas9-mediated gene editing and J.E. performed in vivo experiments. Y.-J.H. analyzed the RNA-seq data. Z.Z. and J.L. performed in vivo experiments.

A.C. and M.H. contributed to vector construction. M.S. designed the study, analyzed and interpreted the data, and wrote the manuscript.

Competing interests

A patent application has been submitted based in part on results presented in this manuscript. J.F., J.S., M.H., and M.S. are listed as the inventors. The Memorial Sloan Kettering Cancer Center has licensed this intellectual property, has received license fees, and has the potential to receive royalties under the license.

Extended data is available for this paper at https://doi.org/10.1038/s41591-018-0290-5.

Supplementary information is available for this paper at https://doi.org/10.1038/s41591-018-0290-5.

Publisher's note: Springer Nature remains neutral with regard to jurisdictional claims in published maps and institutional affiliations. Data availability

The RNA-seq data have been deposited in the Gene Expression Omnibus and are available under accession number GSE121226. Raw data for the figures in the manuscript will be made available upon request to the corresponding author.

Online content

Any methods, additional references, Nature Research reporting summaries, source data, statements of data availability and associated accession codes are available at https://doi.org/10.1038/s41591-018-0290-5.

Reporting Summary. Further information on research design is available in the Nature Research Reporting Summary linked to this article.
} 
exhaustion ${ }^{9,10}$, which may be accentuated by the redundancy of $\mathrm{CD} 28$ and $\mathrm{CD} 3 \zeta$ signaling ${ }^{11,12}$ as well as the spatiotemporal constraints imparted by the structure of second-generation CARs ${ }^{2}$. Thus, we hypothesized that calibrating the activation potential of CD28-based CARs would differentially reprogram $\mathrm{T}$ cell function and differentiation. Here, we show that CARs encoding a single immunoreceptor tyrosine-based activation motif direct $\mathrm{T}$ cells to different fates by balancing effector and memory programs, thereby yielding CAR designs with enhanced therapeutic profiles.

We hypothesized that the redundancy of $\mathrm{CD} 28$ and $\mathrm{CD} 3 \zeta$ signaling in a chimeric antigen receptor (CAR) design incorporating all three $\mathrm{CD} 3 \zeta$ immunoreceptor tyrosine-based activation motifs (ITAMs) ${ }^{11,13}$ may foster counterproductive $\mathrm{T}$ cell differentiation and exhaustion ${ }^{9,10}$. Therefore, we calibrated ITAM activity by mutating tyrosine residues to impede their phosphorylation and downstream signaling ${ }^{14-17}$. To investigate the contribution of each of the three CD3 5 ITAMs to the function, differentiation, and therapeutic potency of $1928 \zeta$-engineered T cells, we generated single iTAM-containing $1928 \zeta$ mutants termed 1XX, X2X, and XX3 (Fig. 1a). In one additional mutant, termed X23, we retained the two distal ITAMs (ITAM2 and ITAM3), both of which have been shown to display lower binding affinity for tyrosine-protein kinase ZAP-70 relative to ITAM $1^{13,18}$. All mutant CARs were similarly expressed in retrovirally transduced human peripheral blood T cells (Fig. 1b); they were found to direct indistinguishable cytotoxicity in a 4-h chromium-51 $\left({ }^{51} \mathrm{Cr}\right)$ release assay and proliferation in response to three weekly stimulations with CD19 antigen (Extended Data Fig. 1a,b).

Remarkably, the therapeutic efficacy of CAR T cells expressing these mutant CARs differed singularly in the well-established pre-B acute lymphoblastic leukemia Nalm6 mouse model ${ }^{8,19,20}$. CAR $\mathrm{T}$ cell doses were deliberately lowered to better compare $\mathrm{T}$ cell potency, as previously described in the CAR stress test ${ }^{8}$. Relative to the parental 1928C, XX3 showed markedly diminished antitumor efficacy, only achieving a transient reduction of tumor burden, whereas 1XX exceeded 19285, inducing long-term remission in all mice (Fig. 1c and Extended Data Fig. 1c). Treatment with X2X and X23 did not significantly alter survival rates compared to $1928 \zeta$ ( $1928 \zeta$ versus X2X: $P=0.6942 ; 1928 \zeta$, versus X23: $P=0.1085$ ).

Thus, while the presence of one (1XX, X2X, XX3), two (X23), or three (1928 functional ITAMs did not noticeably alter short-term in vitro function, a single ITAM-containing CAR outperformed the triple- and double-ITAM-containing CARs in vivo. Efficacy of tumor eradication gradually decreased with increasing distal positioning of the functional ITAM. The 1XX CAR consistently showed rapid tumor eradication and was the only CAR design to achieve durable and complete remissions at the lowest $\mathrm{T}$ cell dose. Treatment with X2X slightly delayed tumor progression compared to wild-type 1928 $\mathrm{\zeta}$, but relapses eventually developed. The XX3 CAR did not achieve any tumor control, leading to rapid tumor progression and significantly reduced survival (Fig.1c and Extended Data Fig. 1c).

To investigate the functional basis for these major differences in antitumor efficacy, we examined the responses imparted by these CARs in greater depth. In prolonged (18-h) cytotoxicity assays at a low effector-to-target ratio, XX3 proved to be less active than $1928 \zeta$ and the other mutants (Extended Data Fig. 2a). The diminished effector function of XX3 was further corroborated by reduced granzyme B (GrB) expression (Extended Data Fig. 2b) 
and decreased single and polyfunctional type $1 \mathrm{~T}$ helper cell $\left(\mathrm{T}_{\mathrm{H}} 1\right)$ cytokine secretion (Extended Data Fig. 2c) following stimulation with $\mathrm{CD} 19^{+}$targets. Inclusion of a single ITAM (either ITAM1, 2, or 3) limited T cell differentiation as determined by CD62L/ CD45RA expression, resulting in an increased fraction of central memory CAR T cells and a reduced proportion of effector cells in response to repeated in vitro stimulation with CD19+ targets (Extended Data Fig. 1d).

These findings were extended in vivo as CAR T cells with two inactive CD3 $\zeta$ ITAM domains exhibited increased persistence (Fig. 1d) and delayed T cell differentiation (Fig. 1e). $1 \mathrm{XX}, \mathrm{X} 2 \mathrm{X}$, and XX3 CAR T cells all demonstrated a higher percentage of CD62L ${ }^{+} \mathrm{CD} 45 \mathrm{RA}^{-}$central memory $\mathrm{T}$ cells $\left(\mathrm{T}_{\mathrm{CM}}\right)$ and a decrease in the fraction of terminally differentiated CD62L ${ }^{-} \mathrm{CD} 45 \mathrm{RA}^{+}$effector cells $\left(\mathrm{T}_{\mathrm{EFF}}\right.$ ) (Fig. 1e and Extended Data Fig. 1e). The attenuation of effector differentiation in $\mathrm{CD} 4^{+}$and $\mathrm{CD} 8^{+} \mathrm{CAR} \mathrm{T}$ cells was associated with increased accumulation of both $\mathrm{T}$ cell subsets at the tumor sites (Fig. 1d), establishing the benefit of reducing CD3 $\zeta$ signaling within CD28-based, second-generation CARs.

We next investigated whether the lesser potency of XX3 is intrinsic to ITAM3 or due to its distal position. Therefore, we generated $1928 \zeta$ mutant CARs with either ITAM1 or ITAM3 at the same proximal CAR position through deletion of the remaining CD3 $\zeta$ chain (D12 and D23, Fig. 2a,b). In vitro studies demonstrated equipotent cytolytic activity between all constructs in 4- and 18-h assays, undiminished cytokine and GrB secretion, and comparable proliferative potential between D12, D23, and 1928S (Extended Data Figs. 3 and 4). However, D12 and D23 both outperformed $1928 \zeta$ in vivo, achieving complete tumor eradication (Fig. 2c and Extended Data Fig. 3d). Both CAR constructs moderately diminished $\mathrm{T}$ cell differentiation and promoted higher CAR $\mathrm{T}$ cell accumulation at the tumor site relative to $1928 \zeta$ (Fig. 2d,e).

These findings show the importance of ITAM dosage and position within $1928 \zeta$ CARs. A single functional ITAM is sufficient for potent antitumor efficacy and superior to that afforded by the triple-ITAM-containing wild-type $\mathrm{CD} 3 \zeta$ chain. Furthermore, the therapeutic potency imparted by ITAM3 is less than that of ITAM1 in their respective native positions (XX3 versus 1XX), but comparable at the same proximal position (D12 versus D23).

Despite sharing the same ITAM sequence, D12 achieved superior tumor eradication relative to XX3, demonstrating the importance of ITAM location within second-generation CARs.

To rule out potentially confounding effects arising from subtly different CAR expression levels, we targeted the CAR complementary DNAs (cDNAs) to the TRAC locus ${ }^{19}$, thereby normalizing transgene regulation and copy number while simultaneously removing the endogenous T cell receptor (TCR; Extended Data Fig. 5a). Under these conditions, 1XX CAR T cells again surpassed those expressing $1928 \zeta$ or XX3 (Fig. 3a). TRAC-1XX T cells achieved complete remissions within $21 \mathrm{~d}$, while TRAC-XX3 T cells only delayed tumor progression and could not achieve tumor control (Fig. 3a). Analyses of CAR T cells and tumor cells retrieved $17 \mathrm{~d}$ post-CAR infusion showed higher accumulation of TRAC-1XX and TRAC-XX3 CAR T cells compared to TRAC-1928 in both $\mathrm{CD} 4^{+}$and $\mathrm{CD} 8^{+}$subsets (Fig. 3c and Extended Data Fig. 5b,e). Increased CAR T cell persistence was associated with a higher percentage of $\mathrm{T}_{\mathrm{CM}}$ and interleukin-7 receptor (IL7R) ${ }^{+} \mathrm{CAR} \mathrm{T}$ cell counts 
(Extended Data Figs. 5c,d), reinforcing our findings of delayed T cell differentiation in $1928 \zeta$ mutants. Similar to retrovirally engineered T cells, TRAC-XX3 T cells lost their cytolytic potential over time in contrast to TRAC-1928 and TRAC-1XX cells (Extended Data Fig. 5f). The expression of exhaustion markers was reduced in $1928 \zeta$ mutants compared to $T R A C-1928 \zeta$, and functional exhaustion of $T R A C-1928 \zeta$ was confirmed by reduced $\mathrm{T}_{\mathrm{H}} 1$ cytokine secretion and $\mathrm{GrB} / \mathrm{CD} 107$ a expression after $\mathrm{CAR}$ isolation from treated mice and ex vivo re-exposure to Nalm6 cells (Fig. 3d and Extended Data Fig. 6). Whereas $T R A C-1 \mathrm{XX}$ retained high cytolytic function and the capacity of coproducing multiple cytokines, TRAC-1928 displayed progressive inability to secrete $\mathrm{T}_{\mathrm{H}} 1$ cytokines associated with increased expression of exhaustion markers.

Long-term follow-up studies after administration of two different $\mathrm{T}$ cell doses $\left(5 \times 10^{5}\right.$ and 1 $\times 10^{5} \mathrm{CAR}^{+} \mathrm{T}$ cells) unequivocally demonstrated the therapeutic superiority of TRAC-1XX. While tumor-related deaths occurred within less than $60 \mathrm{~d}$ (treatment with $5 \times 10^{5} \mathrm{CAR}^{+} \mathrm{T}$ cells) or less than $40 \mathrm{~d}$ (treatment with $1 \times 10^{5} \mathrm{CAR}^{+} \mathrm{T}$ cells), all mice treated with TRAC-1XX showed complete tumor eradication and enhanced survival throughout the observation period (Fig. 3b and Extended Data Fig. 10).

The enhanced ability of $1 \mathrm{XX}$ to develop into highly functional, long-lived memory cells was further demonstrated when sorted naive $\mathrm{T}$ cells were used for in vivo therapy. TRAC-1XX showed an increase in the population of $\mathrm{T}_{\mathrm{CM}}$ and IL7R-expressing cells over time (Extended Data Fig. 7a-c), associated with enhanced persistence of highly potent CAR T cells. Importantly, TRAC-1XX CARs were capable of eliciting effective recall responses, achieving complete tumor control following tumor rechallenge (Fig. 3e). Persisting 1XX CAR $\mathrm{T}$ cells comprised increased numbers of $\mathrm{T}_{\mathrm{CM}}, \mathrm{T}_{\mathrm{EFF}}$, and IL7R ${ }^{+}$CARs (Figs. 3f, $\mathrm{g}$ and Extended Data Fig. 7d-f). In contrast, TRAC-1928 more readily acquired an exhausted phenotype (Fig. 3h and Extended Data Fig. 7g) accompanied by reduced CAR T cell persistence without memory formation and the inability to control tumor rechallenge.

To further characterize these different phenotypic and functional patterns, we compared the

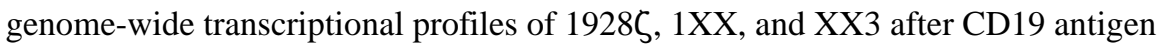
stimulation of TRAC-edited naive T cells. Principal component analysis demonstrated distinct clustering of CAR T cells dependent on their CD3 $\zeta$ mutation (Extended Data Fig. 8a). Consistent with functional studies, major differences emerged in transcriptomic profiles related to $\mathrm{T}$ cell function, differentiation, and exhaustion. Gene set enrichment analysis (GSEA) revealed downregulation of naive/memory-associated genes and enrichment of $\mathrm{T}$ cell activation- and effector-related genes in 1928 $\zeta$ relative to both XX3 and 1XX (Fig. 4a and Extended Data Fig. 8b). As expected, 1928 C CAR T cells showed the greatest upregulation of $\mathrm{T}$ cell differentiation, exhaustion, and apoptosis gene expression profiles (Fig. 4b,c and Extended data Fig. 9a). Gene ontology and GSEA analyses identified significant upregulation of the gene sets associated with inflammation, cytokine, and chemokine activity in $1928 \zeta$ compared to XX3 and in $1 \mathrm{XX}$ compared to XX3 (Fig. 4c and Extended Data Fig. 9b,c). These gene sets were also enriched in 1928 5 relative to 1XX, albeit to a lesser extent, consistent with the intermediate status of $1 X X$ relative to $1928 \zeta$ and XX3 (Fig. 4b,c and Extended Data Fig. 9b,c). To compare TRAC-edited CAR T cells to the effector and memory features of nongenetically modified $\mathrm{CD} 8^{+} \mathrm{T}$ cell subsets, we sorted 
naive $\left(\mathrm{T}_{\mathrm{N}}\right)$, stem cell memory $\left(\mathrm{T}_{\mathrm{SCM}}\right)$, and $\mathrm{T}_{\mathrm{EFF}}$ from resting peripheral blood mononuclear cells as their characteristic transcriptional profiles were previously determined ${ }^{21}$ (Extended Data Fig. 8c). Differentially expressed genes (adjusted $P<0.05$ ) associated with effector versus naive/memory $\mathrm{T}$ cell fate distinguished these CAR constructs (Fig. 4b). Interestingly, $T R A C-1 \mathrm{XX}$ cells exhibited more similarity to $\mathrm{T}_{\mathrm{SCM}}$ than $T R A C-1928 \zeta$ and TRAC-XX3 T cells, which in turn were more similar to $\mathrm{T}_{\mathrm{EFF}}$ and $\mathrm{T}_{\mathrm{N}}$ cells, respectively (Fig. $4 \mathrm{~b}$ ).

Upregulation of key regulators of effector differentiation such as T-bet (and T-bet:Eomes ratio), PR domain zinc finger protein 1 (PRDM1), and DNA-binding protein inhibitor ID-2 (refs. ${ }^{22-24}$ ) with concurrent loss of naive/memory-associated markers (for example, CCR7, CD27, and IL7R ${ }^{21}$ ) corroborated the rapid acquisition of an effector state seen with $1928 \zeta$ (Fig. 4b and Extended Data Fig. 9c,d). In contrast, the tuned signaling strength in 1XX resulted in decelerated $\mathrm{T}$ cell differentiation and preserved expression of important memoryassociated transcription factors, such as transcription factor 7 (TCF7), B-cell lymphoma 6 protein (BCL6), lymphoid enhancer-binding factor 1 (LEF1), and Krüppel-like factor 2 $(\mathrm{KLF} 2)^{23-26}$ (Fig. 4b), further reflected in the upregulation of KLF2-regulated genes such as CCR7, CD62L, ITGB7, and S1PR1 (refs. ${ }^{27,28}$ ) (Fig. 4b and Extended Data Fig. 9d). While $1 \mathrm{XX}$ T cells only demonstrated a partial shift toward a less-differentiated T cell state, XX3 revealed high levels of naive/memory-associated genes together with a systematic suppression of genes encoding master transcription factors for $\mathrm{T}$ cell differentiation and effector function (Fig. 4b-d). These findings underscore the critical role of ITAM dosage and position to impart different $\mathrm{T}$ cell fates. They further support models of memory formation that place cell fate under the control of strength of signaling ${ }^{24,29}$.

Recent reports support the notion that less-differentiated $\mathrm{T}$ cell subsets and memory features are associated with increased antitumor potency and persistence of adoptively transferred $\mathrm{T}$ cells $^{30,31}$. Our results extend these findings in demonstrating that $\mathrm{T}$ cell differentiation states can be intrinsically controlled by mutations in the CD3 $\zeta$ chain of CAR T cells. The CD28 and $\mathrm{CD} 3 \zeta$ signaling domains are constrained into a 1:1 stoichiometry within CARs ${ }^{2}$, differing from their natural spatiotemporal relationship ${ }^{11}$. Prior studies on $\mathrm{CD} 3 \zeta$ mutations were limited to in vitro comparisons ${ }^{32-34}$ that did not uncover major effects, in contrast to the crucial consequences we uncovered in vivo (Fig. 1). Our in vivo studies demonstrate that balancing $\mathrm{T}$ cell differentiation and acquisition of effector functions is essential to optimize therapeutic potency of CAR T cells based on a comparison of different signaling motifs expressed from a constant genomic locus (Figs. 3 and 4). We believe this experimental setting to be optimal for quantitative CAR and TCR comparisons ${ }^{19}$.

In summary, 1XX induced strong effector functions without shutting off memory programs and outperformed $1928 \zeta$ in stress test conditions. Further reducing T cell activation potential through XX3 resulted in highly persisting $\mathrm{T}$ cells but mediocre antitumor activity owing to insufficient elicitation of effector functions. Remarkably, the modified ITAM configuration in 1XX favors persistence of highly functional CARs, balancing the replicative capacity of long-lived memory cells and the acquisition of effective antitumor function. Preparations for clinical studies that utilize the 1XX CAR design are underway. 


\section{Methods}

\section{Cells lines and culture conditions.}

Nalm6 cells were transduced to express firefly luciferase (FFLuc)-green fluorescent protein (GFP), and murine thymoma EL4 cells and NIH/3T3 cells were transduced to express human CD19 as described previously ${ }^{8,20}$. Cells were regularly tested for Mycoplasma using the MycoAlert Mycoplasma Detection Kit (Lonza) and found to be negative.

\section{Isolation and expansion of human T cells.}

Buffy coats from anonymous healthy donors were purchased from the New York Blood Center (institutional review board-exempted) and peripheral blood was obtained from healthy volunteers. All blood samples were handled following the required ethical and safety procedures. Peripheral blood mononuclear cells were isolated by density gradient centrifugation and activated with phytohemagglutinin $\left(2 \mu \mathrm{g} \mathrm{m}{ }^{-1}\right)$ for experiments performed with retroviral vectors or T cells were purified using the Pan T Cell Isolation Kit (Miltenyi Biotec) and stimulated with CD3/CD28 T cell Activator Dynabeads (Invitrogen) as described previously ${ }^{19}$ and cultured in X-VIVO 15 Serum-free Hematopoietic Cell Medium (Lonza), supplemented with 5\% human serum (Gemini Bio-Products), $5 \mathrm{ng} \mathrm{ml}^{-1}$ interleukin-7 (IL7) and $5 \mathrm{ng} \mathrm{ml}^{-1}$ IL15 (BioLegend) for the gene targeting experiments. The medium was changed every $2 \mathrm{~d}$, and cells were plated at $10^{6}$ cells per ml.

For some experiments, naive $\left(\mathrm{TCR}^{+} \mathrm{CD} 62 \mathrm{~L}^{+} \mathrm{CCR} 7^{+} \mathrm{CD} 45 \mathrm{RA}^{+} \mathrm{CD} 95^{-}\right), \mathrm{T}_{\mathrm{SCM}}\left(\mathrm{TCR}^{+} \mathrm{CD} 62 \mathrm{~L}\right.$ ${ }^{+} \mathrm{CCR} 7{ }^{+} \mathrm{CD} 45 \mathrm{RA}^{+} \mathrm{CD}^{+} 5^{+}$), and $\mathrm{T}_{\mathrm{EFF}}$ cells $\left(\mathrm{TCR}^{+} \mathrm{CD} 62 \mathrm{~L}^{-} \mathrm{CCR} 7^{-} \mathrm{CD} 45 \mathrm{RA}^{+} \mathrm{CD} 95^{+}\right.$) were sorted by flow cytometry. The CAR cDNA was then targeted to the TRAClocus of sorted naive $\mathrm{T}$ cells, followed by in vitro culture and TCR-negative purification as described later on. Seven days after gene targeting was performed, T cells were stimulated with 3T3-CD19 for $24 \mathrm{~h}$ for further analysis or injected into tumor-bearing mice.

\section{Genetic modification of T cells.}

Plasmids encoding the SFG $\gamma$ retroviral vector ${ }^{35}$ were prepared using standard molecular biology techniques as described previously ${ }^{20}$. Vesicular stomatitis virus glycoprotein $\mathrm{G}$ (VSV-G) pseudotyped retroviral supernatants derived from transduced gpg29 fibroblasts $(\mathrm{H} 29)$ were used to construct stable retroviral-producing cell lines ${ }^{36}$.

Synthesis of SFG-1928 $\zeta$ has been previously described ${ }^{2,20,37}$; it comprises a single-chain variable fragment specific for the human $\mathrm{CD} 19$, preceded by a CD8a leader peptide and followed by $\mathrm{CD} 28$ hinge-transmembrane-intracellular regions, and CD3 $\zeta$ or ITAM-mutated/ deleted CD3 3 intracellular domains linked to a P2A sequence to induce coexpression of truncated low-affinity nerve growth factor receptor (LNGFR). Standard molecular biology techniques were used to construct the $1928 \zeta$ mutants. Tyrosine-to-phenylalanine point mutations within the ITAMs of the CD3 $\zeta$ chain were introduced to generate $1928 \zeta$ CARs with one or two functional ITAMs. For deletion mutations, the membrane-proximal ITAM sequence (ITAM1) was retained or substituted with the membrane-distal ITAM (ITAM3) sequence, and the remaining $\mathrm{CD} 3 \zeta$ domain was deleted. 48-h after initiating $\mathrm{T}$ cell activation, $\mathrm{T}$ cells were transduced with retroviral supernatants by centrifugation on 
RetroNectin-coated plates (Takara), as described previously. Transduction efficiencies were determined $4 \mathrm{~d}$ later by flow cytometry, and CARs were either injected into tumor-bearing mice or used for in vitro experiments.

Gene targeting experiments were performed as described previously ${ }^{19}$. In short, modified guide RNAs (gRNAs) targeting the first exon of the constant chain of the TRA gene ${ }^{19}$ and Cas9 messenger RNA (mRNA) were synthesized by TriLink BioTechnologies. The pAAVTRAC-1928 5 was cloned based on a pAAV-GFP backbone (Cell Biolabs). The pAAV$T R A C-1928 \zeta$ contains $1.9 \mathrm{~kb}$ of genomic TRAC flanking the gRNA targeting sequences, a self-cleaving P2A peptide in-frame with the first exon of TRAC followed by the $1928 \zeta$ or $1928 \zeta$ mutant CAR as described earlier. The CAR cDNA is followed by the bovine growth hormone polyA signal.

CD3/CD28 beads were magnetically removed $48 \mathrm{~h}$ after initiating $\mathrm{T}$ cell activation. T cells were transfected by electrotransfer of Cas9 mRNA and gRNA using an AgilePulse MAX system (BTX) and recombinant AAV6 donor vector (manufactured by SignaGen Laboratories) was added to the culture following electroporation as described previously ${ }^{19}$. To obtain TCR-negative T cells, TCR-positive T cells were removed from the culture using magnetic biotin-anti-TCRa $\beta$, anti-biotin microbeads, and LS columns (Miltenyi Biotec).

\section{Cytotoxicity assays.}

The cytotoxicity of $\mathrm{T}$ cells transduced with a CAR was determined by standard ${ }^{51} \mathrm{Cr}$ release assays and luciferase-based assays. For ${ }^{51} \mathrm{Cr}$ release assays, EL4 expressing CD19 were used as target cells as described previously ${ }^{38}$. For luciferase-based assays, Nalm6-expressing FFLuc-GFP served as target cells. The effector and tumor target cells were cocultured in triplicates at the indicated effector/target ratio using black-walled 96-well plates with $5 \times$ $10^{4}$ target cells in a total volume of $100 \mu \mathrm{l}$ per well in Nalm6 medium. Target cells alone were plated at the same cell density to determine the maximal luciferase expression (relative light units (RLU)); $18 \mathrm{~h}$ later, $100 \mu \mathrm{l}$ luciferase substrate (Bright-Glo; Promega) was directly added to each well. Emitted light was detected in a luminescence plate reader. Lysis was determined as $(1-($ RLUsample $) /($ RLUmax $)) \times 100$.

\section{Antigen stimulation and proliferation assays.}

Four days after transduction, CAR T cells were cocultured with irradiated confluent CD19+ $\mathrm{NIH} / 3 \mathrm{~T} 3$, at $10^{6} \mathrm{CAR}^{+}$cells per $\mathrm{ml}$ in 24 -well tissue culture plates. Identical stimulations in fresh medium were performed weekly. Total cells were counted and CAR expression was determined weekly by flow cytometry. Subsequently, CAR T cells were restimulated under the same conditions.

\section{Mouse systemic tumor model.}

We used 6- to 12-week-old NOD/SCID/IL-2R $\gamma^{\text {null }}$ male mice (The Jackson Laboratory), under a protocol approved by the Memorial Sloan Kettering Cancer Center (MSKCC) Institutional Animal Care and Use Committee. All relevant animal use guidelines and ethical regulations were followed. Mice were inoculated with $0.5 \times 10^{6}$ FFLuc-GFP NALM6 cells by tail vein injection, followed by $5 \times 10^{4}, 1 \times 10^{5}$, or $5 \times 10^{5}$ CAR T cells injected $4 \mathrm{~d}$ later. 
Tumor rechallenge experiments were performed by intravenous administration of $0.5 \times 10^{6}$, $1 \times 10^{6}$ or $2 \times 10^{6}$ FFLuc-GFP Nalm6 cells at intervals of $7-10 \mathrm{~d}$ at the indicated time points. Nalm6 produce very even tumor burdens, and no mice were excluded before treatment. No randomization or blinding methods were used. Bioluminescence imaging was performed using the IVIS Imaging System (PerkinElmer) with the Living Image software (PerkinElmer) for the acquisition of imaging datasets. Tumor burden was assessed as described previously ${ }^{39}$.

\section{RNA extraction, transcriptome sequencing and RNA-seq analysis.}

Seven days after gene targeting of sorted naive T cells was performed, TRAC-1928 $\zeta$, TRAC-XX3, and TRAC-1XX were stimulated with irradiated 3T3-CD19 for $24 \mathrm{~h}$, followed by magnetic selection of $\mathrm{CD} 8^{+}$cells (Miltenyi Biotec). Washed $\mathrm{T}$ cells were resuspended in $250 \mu \mathrm{l}$ PBS and placed in $750 \mu \mathrm{l}$ TRIzol LS Reagent (Thermo Fisher Scientific). RNA was extracted using the RNeasy Mini Kit (QIAGEN) according to the instructions provided by the manufacturer. After RiboGreen RNA quantification and quality control using an Agilent Bioanalyzer, $500 \mathrm{ng}$ of total RNA underwent polyA selection and TruSeq RNA library preparation according to the instructions provided by the manufacturer (TruSeq Stranded mRNA LT Kit; Illumina), with eight cycles of PCR. Samples were barcoded and run on a HiSeq 4000 (Illumina) in a 50 base pair (bp)/50 bp paired-end run, using the HiSeq 3000/4000 SBS Kit (Illumina). An average of 30.6 million paired reads was generated per sample. At the most, the ribosomal reads represented $4.69 \%$ of the total reads generated; the percentage of mRNA bases averaged $69.3 \%$.

The output FASTQ data were mapped to the target genome using the STAR RNA-seq aligner (version 2.5.0a; two-pass method) ${ }^{40}$, resolving reads across splice junctions. The first mapping pass uses a list of known annotated junctions from Ensemble (https:// www.ensembl.org/index.html). Junctions found during the first pass are then added to the known junctions and a second mapping pass is done using the RemoveNoncanonical flag. After mapping, the output SAM files (SAMtools, version 0.1.19) were processed using PICARD tools (version 1.124; https://broadinstitute.github.io/picard/) to add read groups and AddOrReplaceReadGroups, which in addition sorts the file and converts it to the compressed BAM format. The expression count matrix was then computed from the mapped reads using HTSeq (version 0.5.3; https://htseq.readthedocs.io/en/release_0.10.0/) and one of several possible gene model databases. The $\mathrm{R}$ (version 3.2.0) package DESeq (https:// www.huber.embl.de/users/anders/DESeq/) was used to both normalize the full dataset and analyze differential expression between sample groups.

Differentially expressed genes between $T$ cell subsets $\left(T_{N}, T_{C M}, T_{S C M}, T_{E F F}\right.$ cells) were retrieved from Gattinoni et al. ${ }^{21}$. GSEA was performed using a pre-ranked file generated using $\log _{2}$ (fold change) on curated pathway gene sets from the Broad Institute Molecular Signature Database (http://www.broadinstitute.org/gsea/msigdb/). The gene signatures demonstrated are derived from immunological signatures C7, Reactome, KEGG and gene ontology (Biological Process) gene sets. GSE41867 was used for the GSEA of exhausted versus naive/memory CD8 T cells and to curate the signature of 200 genes upregulated in exhausted CD8 T cells (day 30 post-lymphocytic choriomeningitis virus infection) relative 
to memory and naive cells. Genes identified as differentially expressed between experimental conditions, with an adjusted $P$ value $<0.05$ and absolute $\log _{2}$ (fold change) $>1$, were used for gene ontology analysis using the R package enrichR (http:// amp.pharm.mssm.edu/Enrichr/).

\section{Antibodies and intracellular staining.}

The following fluorophore-conjugated antibodies were used. From BD Biosciences: APCCy7 mouse anti-human CD8; APC-Cy7 mouse anti-human CD45; BUV395 mouse antihuman CD4; PE mouse anti-human CD4; BB515 mouse anti-human CD4; BV421 mouse anti-human CD62L; BV650 mouse anti-human CD45RA; BV421 mouse anti-human CD45RA; BV480 mouse anti-human CD279 (PD-1); BV650 mouse anti-human CD279 (PD-1); BUV737 mouse anti-human CD19; BV421 mouse anti-human TIM3 (CD366); BB515 mouse anti-human CD95; APC-H7 mouse anti-human CD27; PE mouse anti-human CD271 (LNGFR). From BioLegend: APC anti-human CD8a; APC/Cy7 anti-human CD62L; FITC anti-human CD45RA; Brillian Violet 785 anti-human CD366 (Tim-3); PE anti-human CD127 (IL-7Ra); Alexa Fluor 488 anti-human CD127 (IL-7Ra); PerCP/Cyanine5.5 antihuman CD197 (CCR7); Brilliant Violet 650 anti-human CD28. From Invitrogen: CD8a Monoclonal Antibody (RPA-T8), PE-Cyanine7; CD223 (LAG-3) monoclonal antibody (3DS223H) PerCP-eFluor 710; CD223 (LAG-3) Monoclonal Antibody (3DS223H), APC; InvitrogenTCR alpha/beta monoclonal antibody (IP26), PE-Cyanine7. From Miltenyi Biotec: human monoclonal Anti-TCRa/ $\beta$-PE. 7-AAD (BD Biosciences) and LIVE/DEAD Fixable Violet Dead Cell Stain Kit (Invitrogen) were used as viability dyes.

For CAR staining, an Alexa Fluor 647 AffiniPure $\mathrm{F}\left(\mathrm{ab}^{\prime}\right)_{2}$ Fragment Goat Anti-Mouse IgG, $\mathrm{F}\left(\mathrm{ab}^{\prime}\right)_{2}$ fragment specific antibody was used (Jackson ImmunoResearch). For cell counting, CountBright Absolute Counting Beads were added (Invitrogen) according to the manufacturer's instructions. For in vivo experiments, Fc receptors were blocked using FcR Blocking Reagent, mouse (Miltenyi Biotec).

For intracellular cytokine secretion assay, T cells were cocultured with 3T3-CD19 or Nalm6 in the presence of the Golgi Plug protein transport inhibitor (BD Biosciences) for the last 4 h. For CD107a staining, Brilliant Violet 650 anti-human CD107a (LAMP-1) antibody (BioLegend) was added to the coculture. For ex vivo cytokine analyses, CAR T cells were obtained from the bone marrow and spleen of treated mice and purified using a mouse cell depletion kit (Miltenyi Biotec). Samples were processed in biological triplicates (d16) or pooled (due to low cell numbers in $1928 \zeta$-treated mice), and technical triplicates (d36) were performed. Stimulation with phorbol myristate acetate (PMA)/ionomycin (Invitrogen) were performed as the positive control. T cells were subsequently washed, stained for cell surface markers, fixed, and permeabilized according to the manufacturer's instructions using a Cytofix/Cytoperm Fixation/Permeabilization Solution Kit (BD Biosciences). Intracellular cytokine staining was performed with BV421 rat anti-human IL2, BV650 mouse anti-human TNF, BUV395 mouse anti-human TNF, BUV395 mouse anti-human IFN- $\gamma$, or FITC mouse anti-human IFN- $\gamma$ (all BD Biosciences), and APC or PE granzyme B monoclonal antibody (GB12, both Invitrogen). 
Flow cytometry was performed on a LSR II or LSRFortessa instrument (BD Biosciences) and a FACSAria sorter (BD Biosciences) was used for cell sorting. Data were analyzed with the FlowJo software v.10.1 (FlowJo LLC).

\section{Statistical analysis.}

All statistical analyses were performed using the Prism 7 (GraphPad) software. No statistical methods were used to predetermine sample size. Statistical comparisons between two groups were determined by two-tailed parametric or nonparametric (Mann-Whitney $U$-test) $t$-tests for unpaired data or by two-tailed paired Student's $t$-tests for matched samples. For in vivo experiments, the overall survival was depicted by a Kaplan-Meier curve and the log-rank test was used to compare survival differences between the groups. $P$ values $<0.05$ were considered to be statistically significant. The statistical test used for each figure is described in the corresponding figure legend.

\section{Extended Data}


a

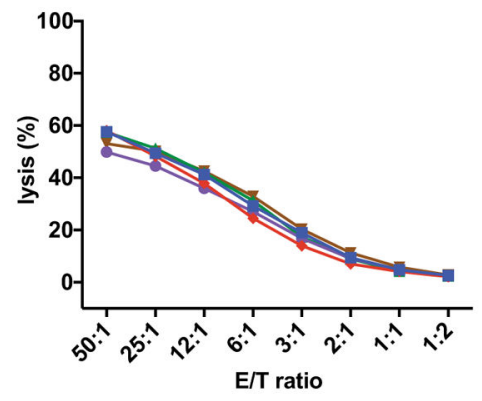

C

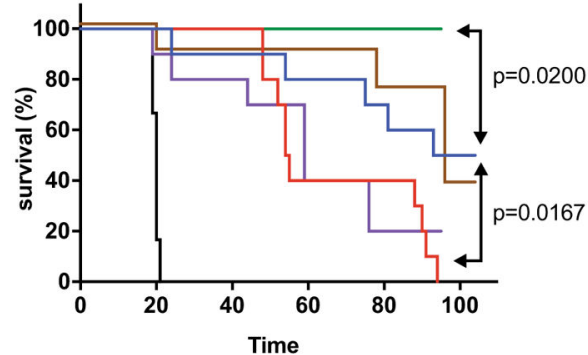

d

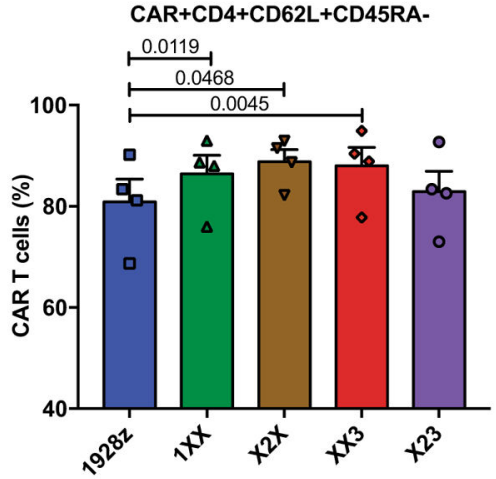

b

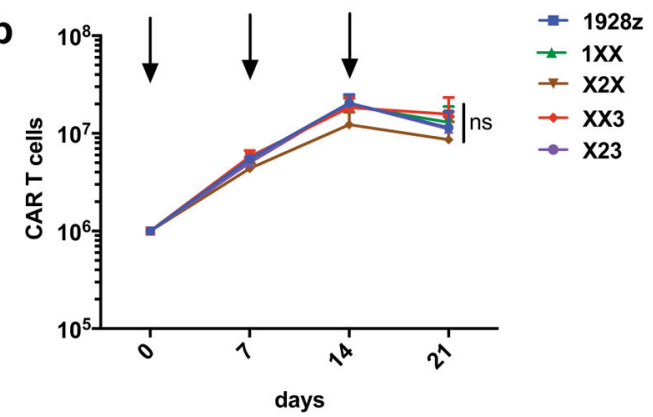

$-1928 z$

$-1 \mathrm{xx}$

$-\mathrm{x} 2 \mathrm{x}$

$-\mathrm{xx3}$

$-\mathrm{x} 23$

$-\mathrm{ctl}$

e

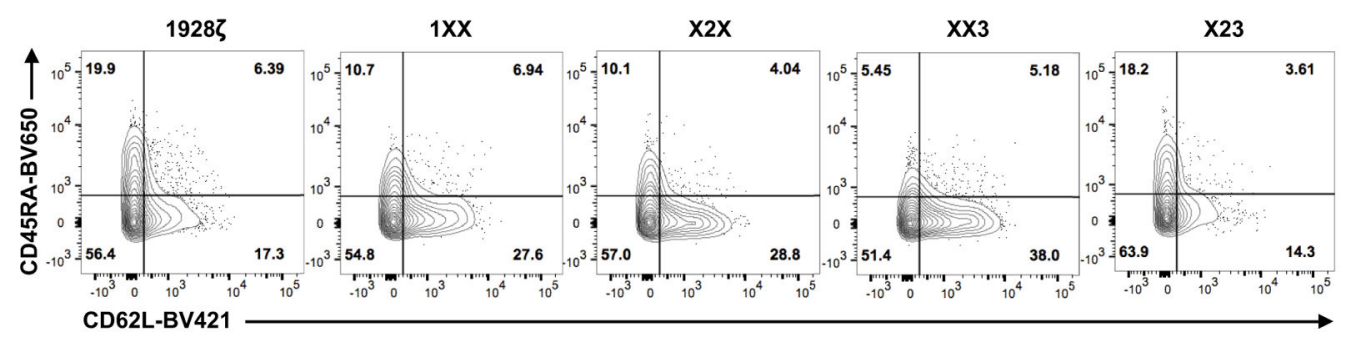

Extended Data Fig. 1 I. Impact of ITAM-mutated 1928 $\zeta$ CARs on $T$ cell function in vitro, $T$ cell differentiation and antitumor activity in vivo.

a, Cytotoxic activity as determined by $4-\mathrm{h}^{51} \mathrm{Cr}$ release assay 1 week after expansion of effector cells on irradiated 3T3-CD19 (data are shown as means of $n=2$ independent experiments performed in triplicates). $\mathbf{b}$, Cumulative cell counts of indicated CAR T cells upon weekly stimulation with $\mathrm{CD} 19^{+}$target cells ( $n=3$ independent experiments). All data are means \pm s.e.m. $P$ values were calculated with two-tailed paired Student's $t$-test. c, NALM6-bearing mice were treated with $5 \times 10^{4} \mathrm{CAR}^{+} \mathrm{T}$ cells. Kaplan-Meier analysis of survival comparing the in vivo efficacy of wild-type $1928 \zeta$ or indicated $1928 \zeta$ mutants $(n=$ 
10 mice, pooled data from two independent experiments). Control (Ctl) refers to untreated mice $(n=6)$. $P$ value was determined by a one-sided log-rank Mantel-Cox test. d, Phenotype of CAR T cells as demonstrated by percentage of central memory (CD62L $\left.{ }^{+} \mathrm{CD} 45 \mathrm{RA}^{-}\right)$and effector memory $\left(\mathrm{CD} 62 \mathrm{~L}^{-} \mathrm{CD} 45 \mathrm{RA}^{-}\right) \mathrm{CD}^{+} \mathrm{CAR} \mathrm{T}$ cells $48 \mathrm{~h}$ upon second stimulation with CD19+ target cells. Two-tailed paired Student's $t$-test was performed, data represent means \pm s.e.m. of $n=4$ independent experiments. e, NALM6-bearing mice were treated with $5 \times 10^{4}$ CAR T cells and euthanized at day 10 after infusion; bone marrow CAR T cells were analyzed by FACS. Representative flow cytometric analysis of phenotype for indicated CAR $\mathrm{T}$ cells as determined by CD62L/CD45RA expression, gated on $\mathrm{CAR}^{+} \mathrm{CD} 4^{+}$ $\mathrm{T}$ cells. Representative of 5 mice per group in at least $n=2$ independent experiments with similar results. 
a

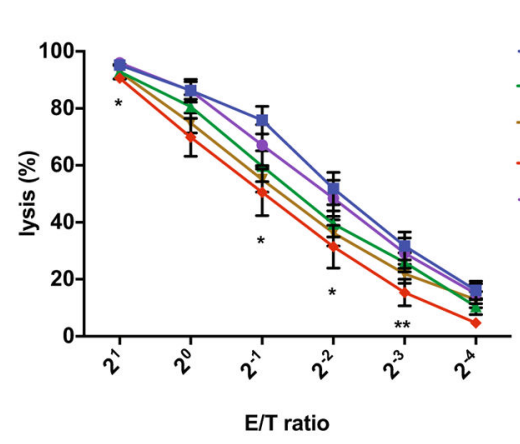

C
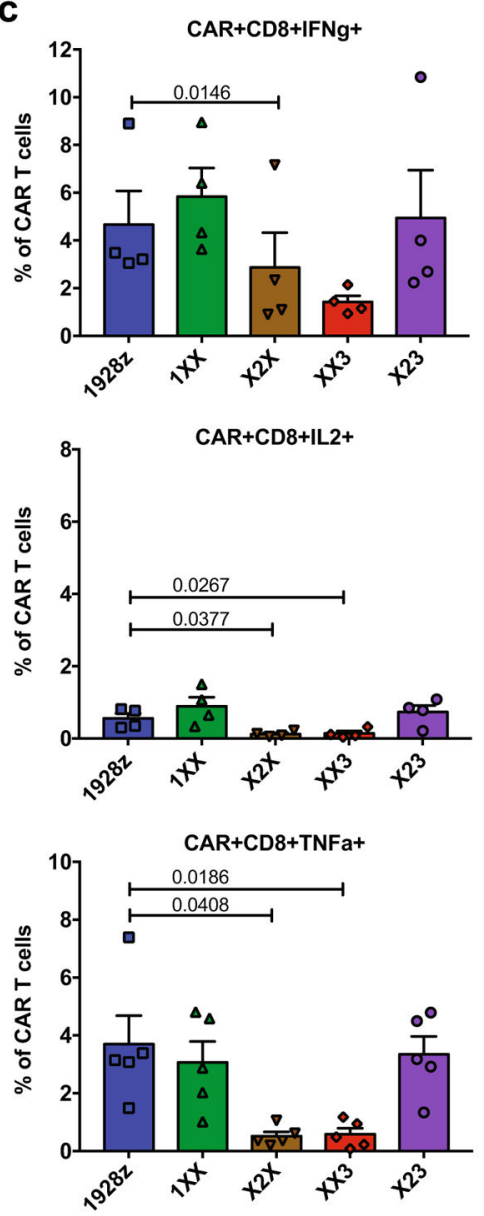

b

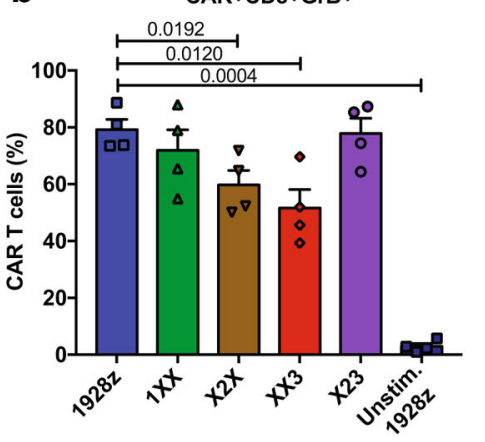

- $1928 z$

- 1XX

$\nabla \quad X 2 X$

- XX3

$\times 23$

- Unstim. 1928z

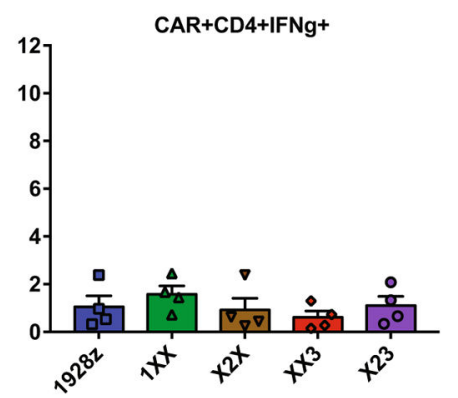

- $1928 z$

- 1XX

$>\quad \times 2 X$

- XX3

- X23
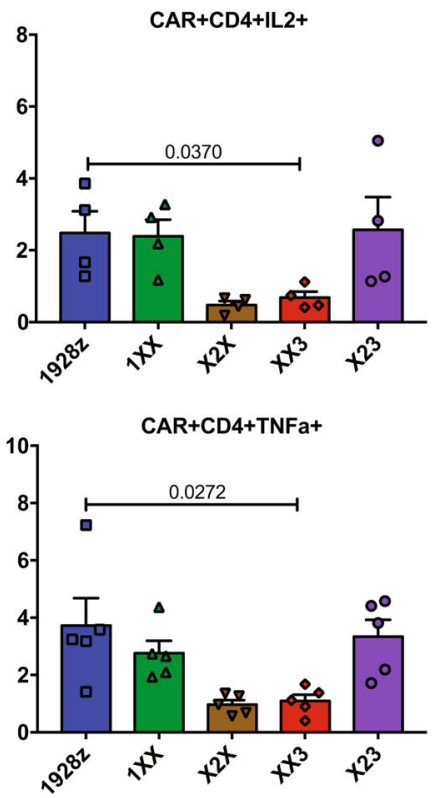

Extended Data Fig. 2 I. Analysis of effector function in $1928 \zeta$ mutants compared to wild-type $1928 \zeta$.

a, Cytotoxic activity of $1928 \zeta$ mutants compared to wild-type $1928 \zeta$ using an 18 -h bioluminescence assay with FFL-expressing NALM6 cells as targets. Experiments were performed 1 week after expansion of effector cells on CD19+ target cells. Data are means \pm s.e.m. ( $n=4$ independent experiments performed in triplicates). $* P<0.05\left(2^{1}: P=0.0273\right.$, $\left.2^{-1}: P=0.0387,2^{-2}: P=0.0125\right), * * P=0.0018$ as calculated by two-tailed paired Student ${ }^{\prime} \mathrm{s}$ $t$-test of average of triplicates. b,c, Granzyme B $(\mathrm{GrB})$ expression $(\mathrm{n}=4$ independent experiments) on $\mathrm{CD}^{+} \mathrm{CAR} \mathrm{T}$ cells (b) and cytokine secretion (c) of $\mathrm{CD}^{+}$and $\mathrm{CD}^{+} \mathrm{CAR}$ 
T cells upon 2nd stimulation with CD19-expressing target cells. All data are means \pm s.e.m. (IFN $\gamma$ and IL2, $n=4$; TNFa, $n=5$ independent experiments). Unstimulated (Unstim.) wildtype $1928 \zeta$ cells were used as control. Significant differences compared to $1928 \zeta$ were determined by two-tailed paired Student's $t$-test. 
a

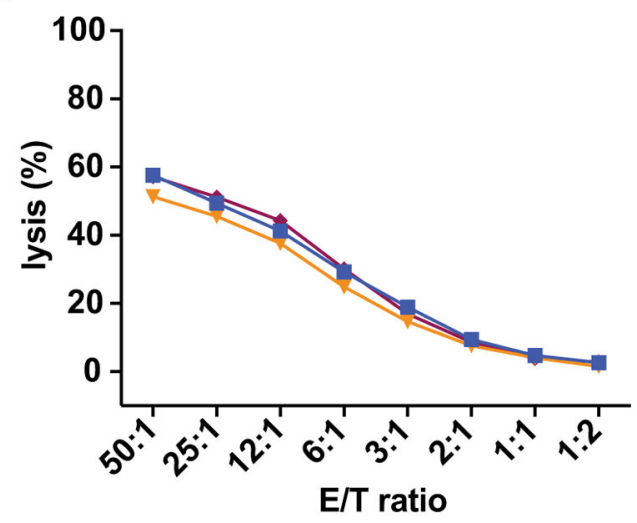

C

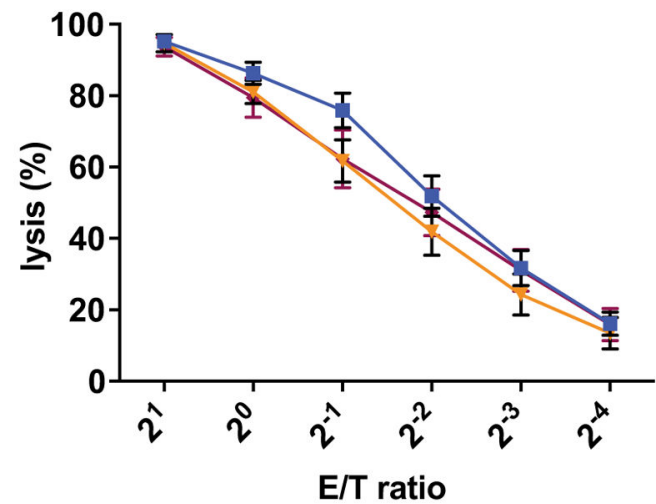

b

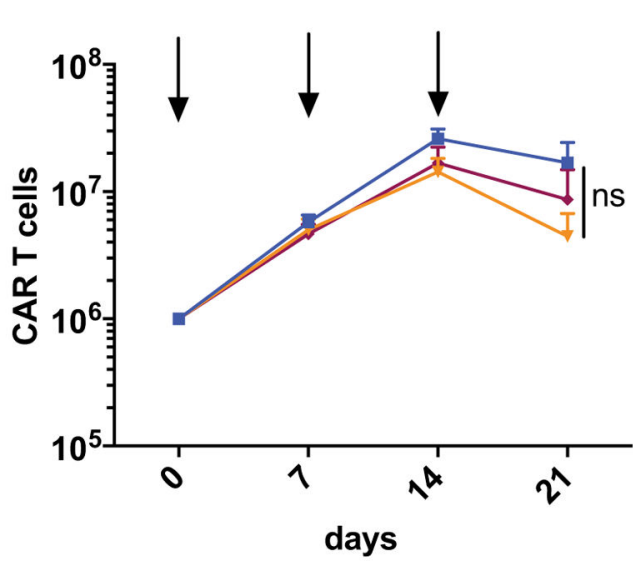

d

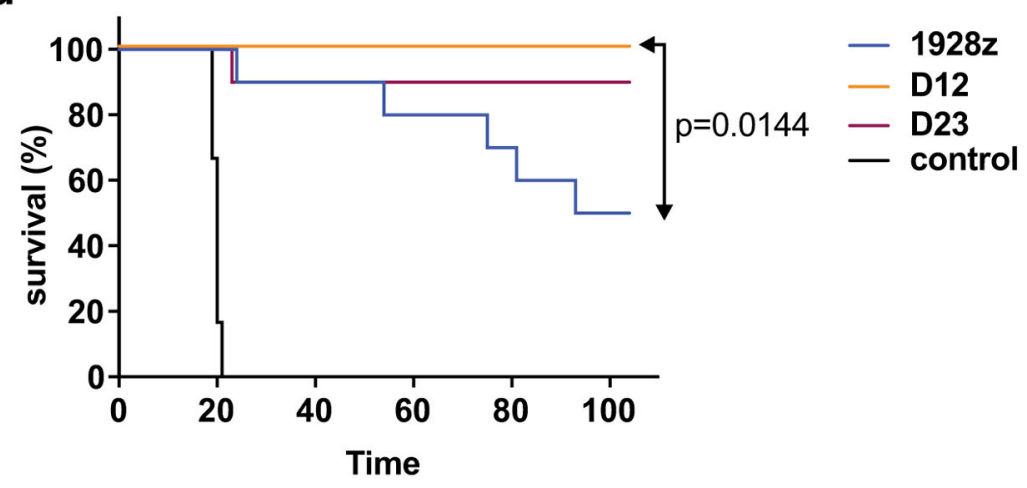

Extended Data Fig. 3 I. Impact of ITAM location within $1928 \zeta$ CARs on T cell function and therapeutic potency.

a, Cytotoxic activity as determined by $4-\mathrm{h}{ }^{51} \mathrm{Cr}$ release assay 1 week after expansion of effector cells on irradiated 3T3-CD19 (data are means of $n=2$ independent experiments performed in triplicates). $\mathbf{b}$, Cumulative cell counts of indicated CAR T cells upon weekly stimulation with $\mathrm{CD} 19^{+}$target cells $(\mathrm{n}=3$ independent experiments). All data are means \pm s.e.m.; $P$ values were calculated with two-tailed paired Student's $t$-test. c, Cytotoxic activity of D12 and D23 compared to wild-type $1928 \zeta$ as determined by 18-h bioluminescence assay with FFL-expressing NALM6 cells as targets. Experiments were performed 1 week after 
expansion of effector cells on CD19 ${ }^{+}$target cells. Data are means \pm s.e.m. $(n=4$ independent experiments performed in triplicates). $P$ value was calculated by two-tailed paired Student ${ }^{\prime} \mathrm{s} t$-test of average of triplicates and showed no significant difference ( $P>$ 0.05 ) between D12/D23 and wild-type $1928 \zeta$ for all E/T ratios. d, NALM6-bearing mice were treated with $5 \times 10^{4}$ CAR T cells. Kaplan-Meier analysis of survival of mice treated with wild-type $1928 \zeta$ or indicated $1928 \zeta$ mutants ( $n=10$ mice per group). Control refers to untreated mice $(n=6)$. $P$ value was calculated by a one-sided log-rank Mantel-Cox test. 
a

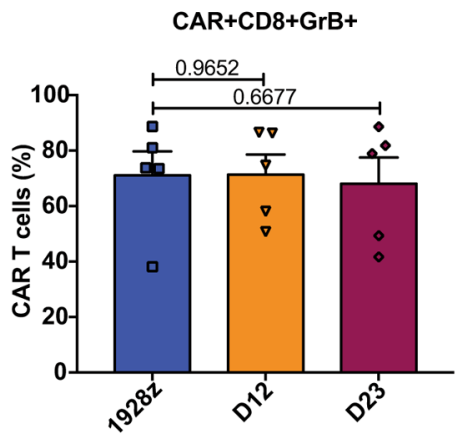

b

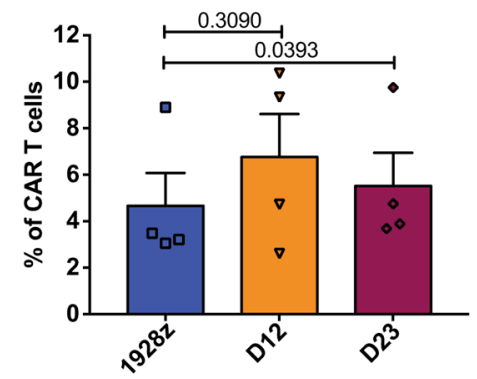

$\mathrm{CAR}+\mathrm{CD} 8+\mathrm{TNFa}+$

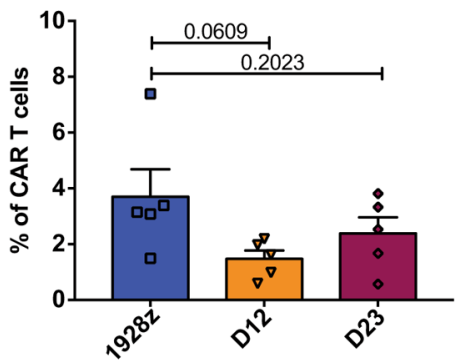

CAR+CD4+IL2+

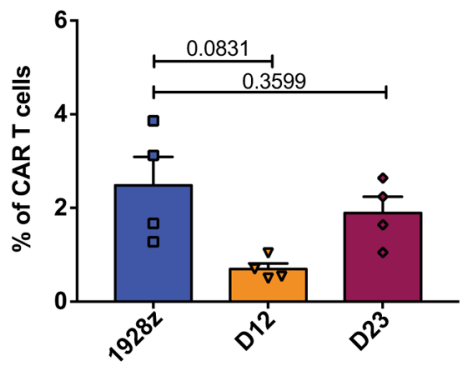

- $1928 \mathrm{z}$

$\checkmark \mathrm{D} 12$

- D23

Extended Data Fig. 4 I. Influence of ITAM location within 1928 $\zeta$ CARs on effector function in vitro.

a, Granzyme B (GrB) expression on $\mathrm{CD}^{+} \mathrm{CAR}$ T cells $(n=5$ independent experiments). b, Cytokine secretion of $\mathrm{CD}^{+}$and $\mathrm{CD}^{+} \mathrm{CAR} \mathrm{T}$ cells upon second stimulation with CD19expressing target cells. Unstimulated wild-type $1928 \zeta$ cells were used as control. All data are means \pm s.e.m. (IFN $\gamma$ and IL2, $n=4$; TNFa, $n=5$ independent experiments). Each individual symbol indicates one sample. Significant differences compared to $1928 \zeta$ were determined by two-tailed paired Student's $t$-test. 
a

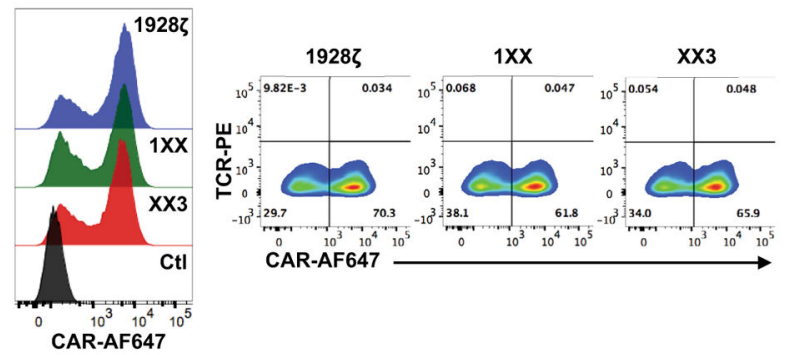

b $\quad \mathrm{CAR}+\mathrm{CD} 4+$

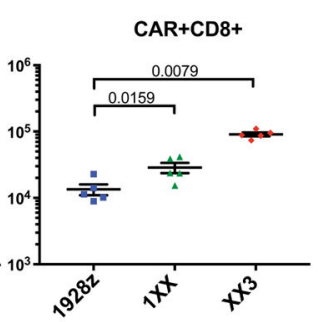

c

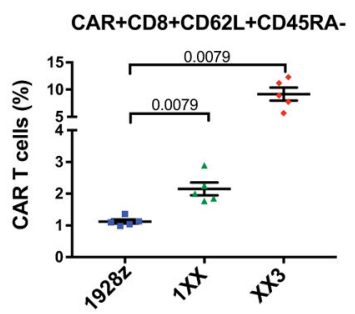

d

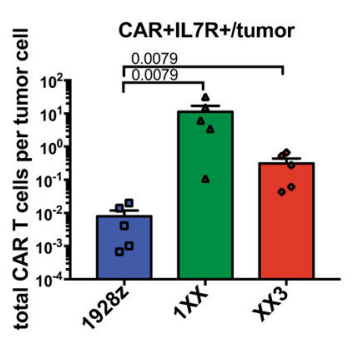

e

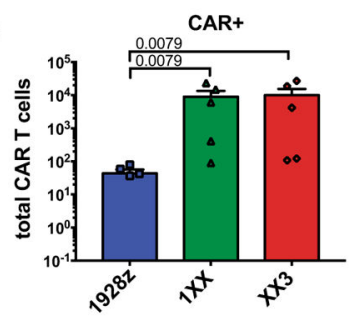

f

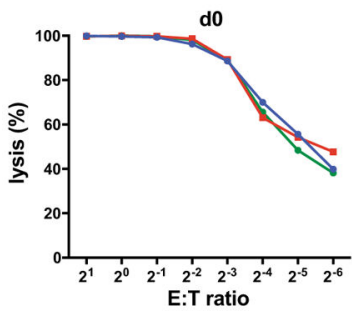

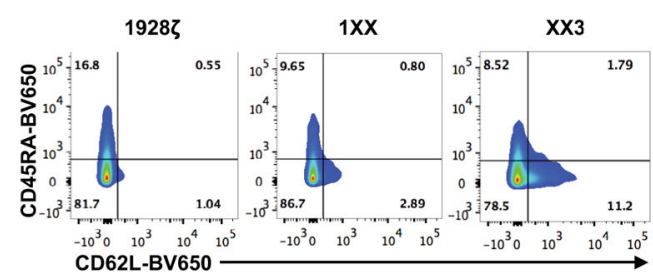
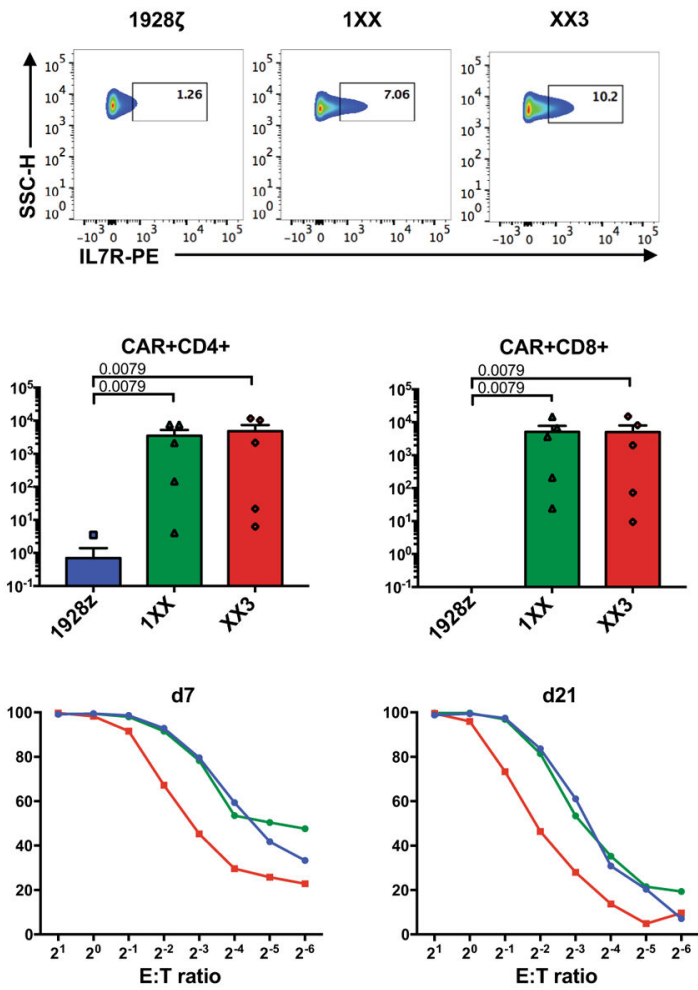

- TRAC-1928z

$\sim$ TRAC-1XX

- TRAC-XX3

Extended Data Fig. 5 I. T cell differentiation and effector function of $T R A C$-encoded $1928 \zeta$ mutants.

NALM6-bearing mice were treated with $1 \times 10^{5} \mathrm{CAR} T$ cells and euthanized at day 17 after infusion. Bone marrow and spleen CAR T cells were analyzed and counted by FACS. a, Histogram and flow cytometric analysis of CAR expression $4 \mathrm{~d}$ after CAR gene integration into the TRAC locus. Representative of four independent experiments with similar results. $\mathrm{b}$, Cell numbers of $\mathrm{CD}^{+}$and $\mathrm{CD} 8^{+} \mathrm{CAR} \mathrm{T}$ cells, $\mathbf{c}$, Percentage of $\mathrm{CD} 8^{+} \mathrm{T}_{\mathrm{CM}}(\mathrm{CD} 62 \mathrm{~L}$

${ }^{+} \mathrm{CD} 45 \mathrm{RA}^{-}$) and flow cytometric analysis of $\mathrm{CD} 62 \mathrm{~L} / \mathrm{CD} 45 \mathrm{RA}$ expression on bone marrow $\mathrm{CD} 8{ }^{+} \mathrm{CAR} T$ cells (representative of $n=5$ mice per group in one independent experiment). 
d, Ratio of CAR ${ }^{+} I L 7 R^{+}$to tumor cells and exemplary flow cytometric analysis of IL7R ${ }^{+}$ CAR T cells in the bone marrow of mice. e, Enumeration of CAR T cells in the spleen of mice. In $\mathbf{b}, \mathbf{c}, \mathbf{d}$ and $\mathbf{e}$ all data are means \pm s.e.m., two-tailed Mann-Whitney analysis was performed, $n=5$ mice per group. f, Cytotoxic activity of TRAC-1XX,TRAC-XX3 and wild-type TRAC-1928C (18-h bioluminescence assay with FFL-expressing NALM6 as targets). Experiments were performed $4 \mathrm{~d}$ post transduction, 1 week and 3 weeks after expansion with weekly CD19 antigen stimulations. Symbols demonstrate means of triplicates (one representative donor). 
a

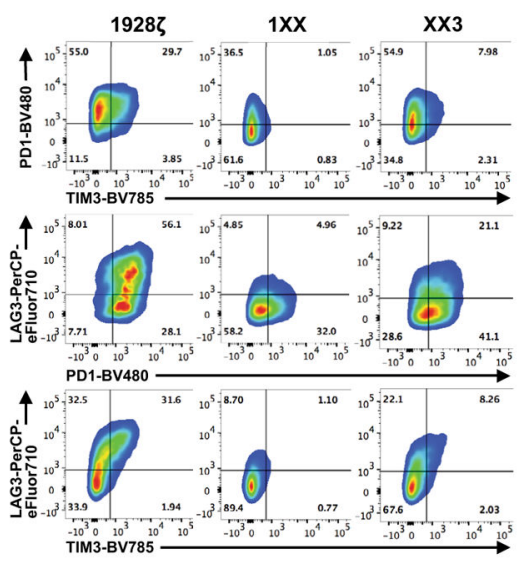

b

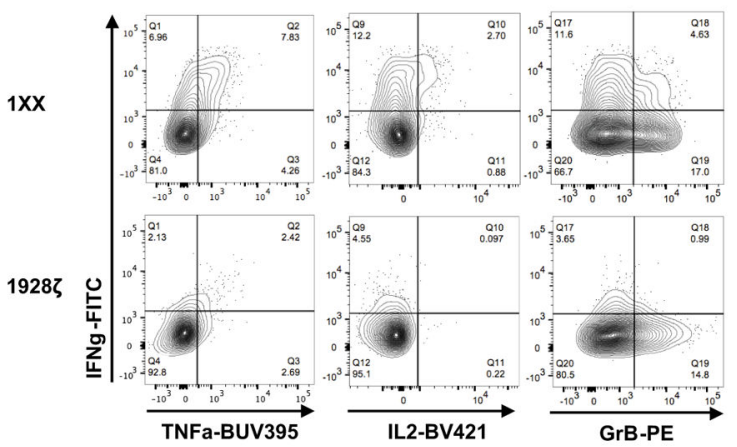

C

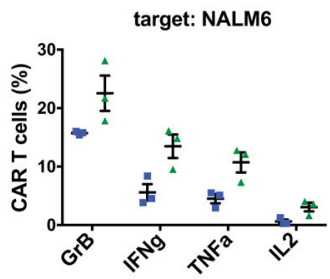

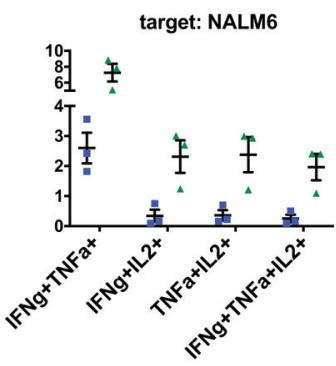

d

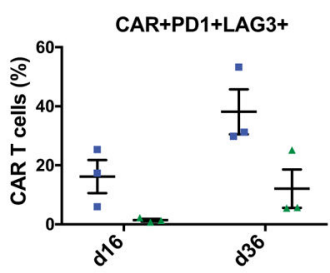

f

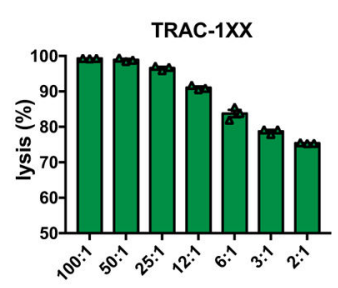

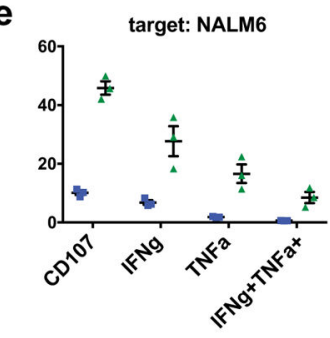

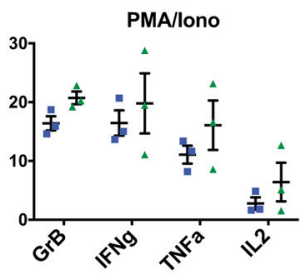

- TRAC-1928z

$\triangle$ TRAC-1XX

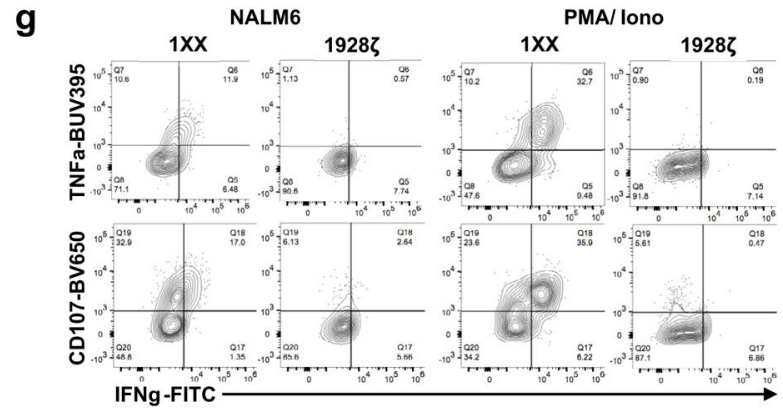

Extended Data Fig. 6 I. In vivo T cell exhaustion of $T R A C$-1928 $\zeta$ mutants compared to wild-type TRAC-1928C.

a, NALM6-bearing mice were treated with $1 \times 10^{5}$ CAR T cells and euthanized at day 17 after infusion. FACS analysis of expression of exhaustion markers on $\mathrm{CAR}^{+} \mathrm{T}$ cells, representative of $n=5$ mice per group in one independent experiment. b-g, NALM6-bearing mice were treated with $1 \times 10^{5} T R A C$-edited naive T cells. $16(\mathbf{b}-\mathbf{c})$ and $36(\mathbf{e}, \mathbf{g}) \mathrm{d}$ after CAR administration, TRAC-1928 and TRAC-1XX cells from bone marrow and spleen were exposed to ex vivo stimulation with NALM6 or PMA/Ionomycin (Iono). Cytokine and granzyme B (GrB)/ CD107a expression on CAR T cells as demonstrated by percentage of 
expression and flow cytometric analyses, representative for $n=3$ mice in two independent experiments (b) and for $n=3$ replicates (g). Expression of exhaustion markers PD1 ${ }^{+} \mathrm{LAG}^{+}$ on CAR T cells (d) and cytotoxic activity (f) of $T R A C$-1XX (day 36) after $10 \mathrm{~h}$ of co-culture with NALM6. All data are means \pm s.e.m., $n=3$ mice per group 

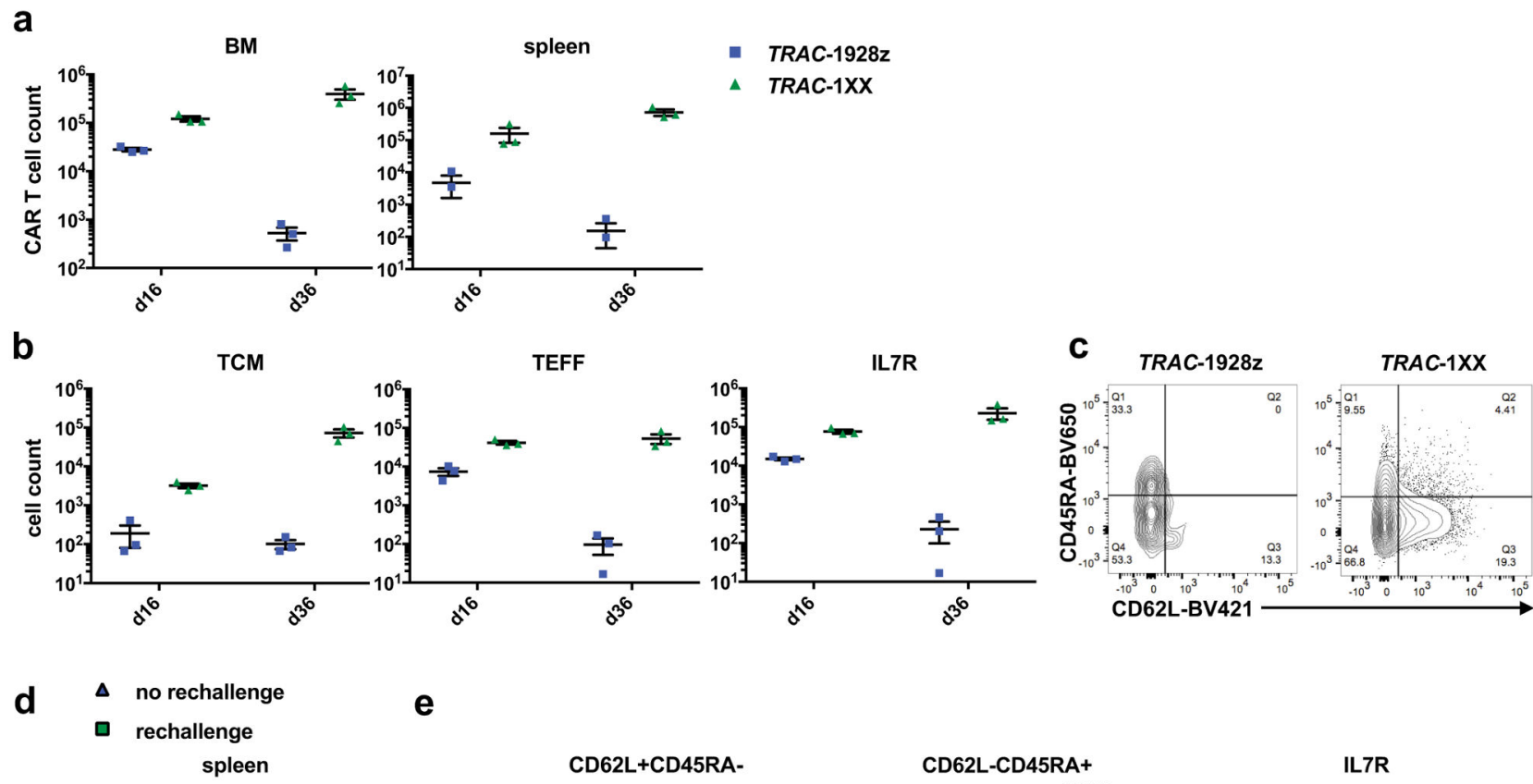

e
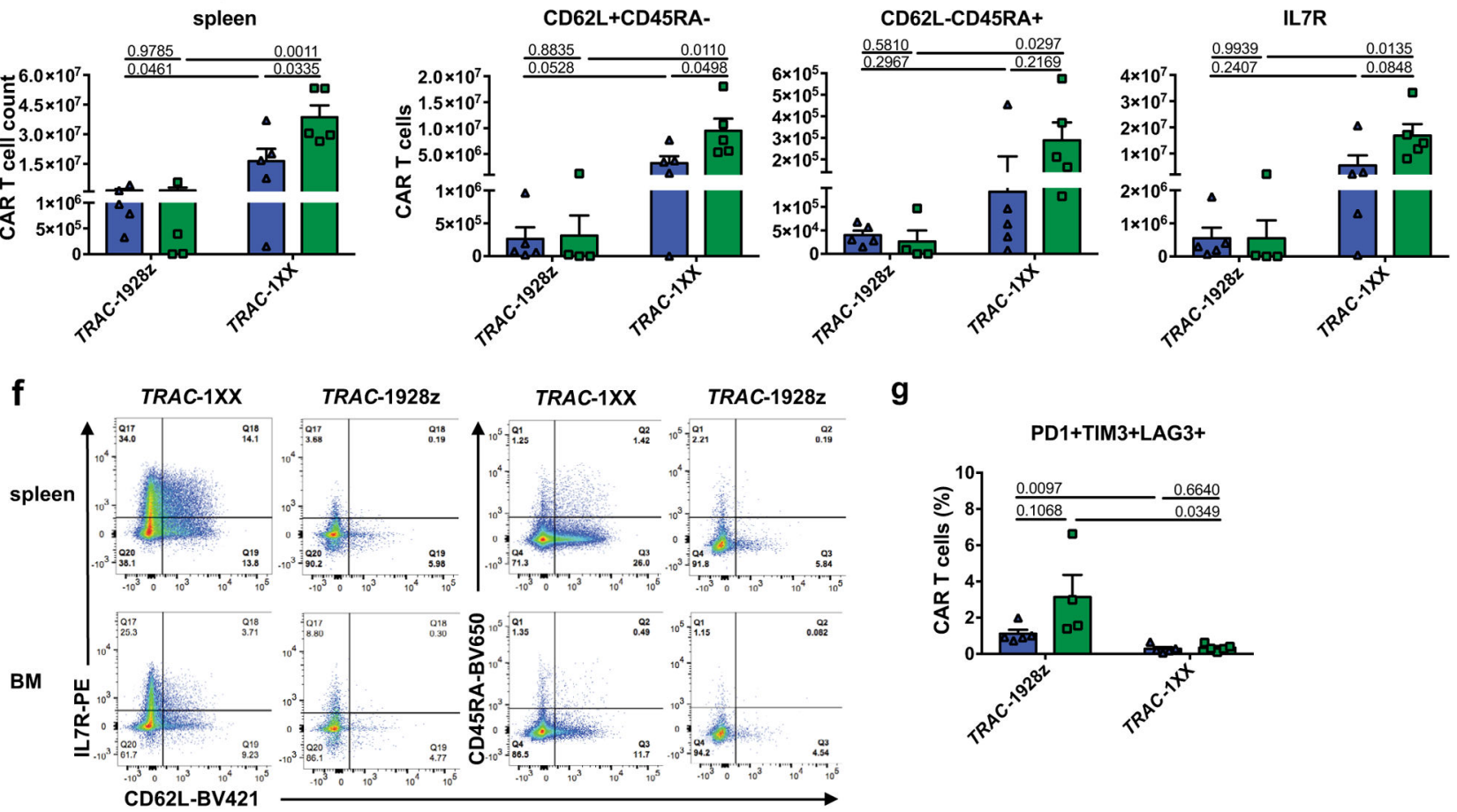

Extended Data Fig. 7 I. T cell memory formation in TRAC-1XX compared to wild-type TRAC-1928C.

NALM6-bearing mice were treated with $1 \times 10^{5}$ or $5 \times 10^{5}$ TRAC-edited naive T cells. a-c, 16 and $36 \mathrm{~d}$ after administration of $1 \times 10^{5} T R A C$-1928 5 and TRAC-1XX, CARs were isolated from bone marrow and spleen. $\mathbf{a}-\mathbf{b}$, Cell number of CAR T cells (a), central memory ( $\mathrm{T}_{\mathrm{CM}}$ : CD62 $\mathrm{L}^{+} \mathrm{CD} 45 \mathrm{RA}^{-}$), effector ( $\mathrm{T}_{\mathrm{EFF}}$ : CD62L-CD45RA $\left.{ }^{+}\right)$and IL7Rexpressing bone marrow CAR T cells $(\mathbf{b})$. All data are means \pm s.e.m., $n=3$ mice per group. c, Representative flow cytometric analysis of CD62L/CD45RA expression on TRAC-1928C and TRAC-1XX bone marrow CAR T cells at day 36 in one independent experiment $(n=3$ 
mice per group). $\mathbf{d}-\mathbf{g}$, NALM6-bearing mice were treated with $5 \times 10^{5}$ TRAC-edited naive T cells and were either rechallenged with NALM6 cells ( $n=5$ mice per group) or no further rechallenge with tumor was performed (TRAC-1928,$n=6$ mice; TRAC-1XX, $n=7$ mice). d,e, Cell number of total CAR T cells (d), $\mathrm{T}_{\mathrm{CM}}, \mathrm{T}_{\mathrm{EFF}}$ and IL7R $\mathrm{R}^{+}$CAR T cells (e) in the spleen of treated mice $63 \mathrm{~d}$ post CAR administration (rechallenge: TRAC-1928,$n=4$ mice; TRAC-1XX, $n=5$ mice. No rechallenge, $n=5$ mice per group). All data are means \pm s.e.m.; a two-tailed unpaired Student's $t$-test was used for statistical analysis. f, FACS analysis of $\mathrm{IL}^{+} \mathrm{R}^{+}, \mathrm{CD}_{2} \mathrm{~L}^{+}$and $\mathrm{CD} 45 \mathrm{RA}^{+}$expression on TRAC-1928C and TRAC-1XX CAR T cells at day 63 post CAR infusion (representative for at least $n=3$ mice per group in one independent experiment). g, Expression of exhaustion markers PD ${ }^{+} \mathrm{TIM}^{+}{ }^{+} \mathrm{LAG} 3^{+}$on CAR T cells derived from the spleen (rechallenge: TRAC-1928C, $\mathrm{n}=4$ mice; TRAC-1XX: $n$ $=5$ mice. No rechallenge, $n=5$ mice per group). All data are means \pm s.e.m.; $P$ value was determined by a two-tailed unpaired Student's $t$-test. 
a

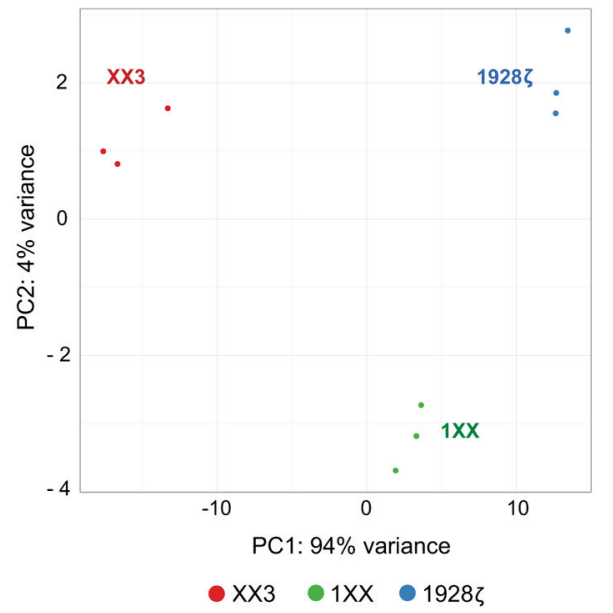

b
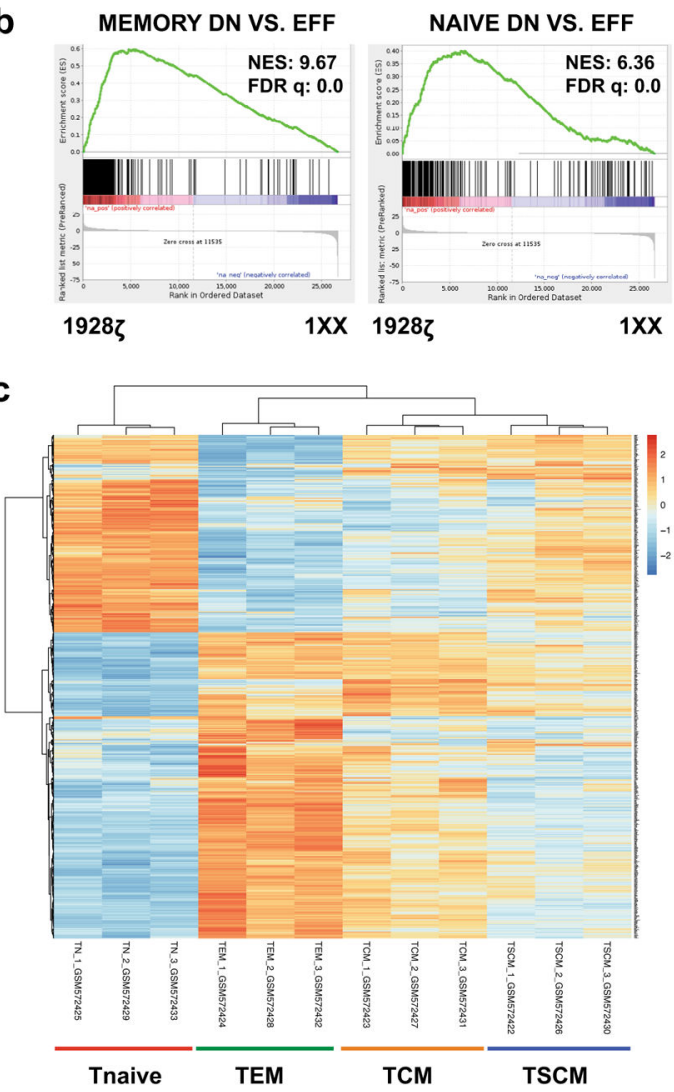

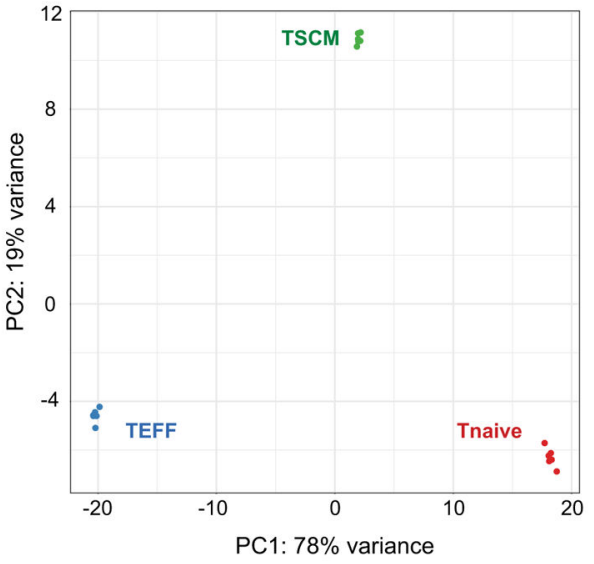

- Tnaive TSCM TEFF
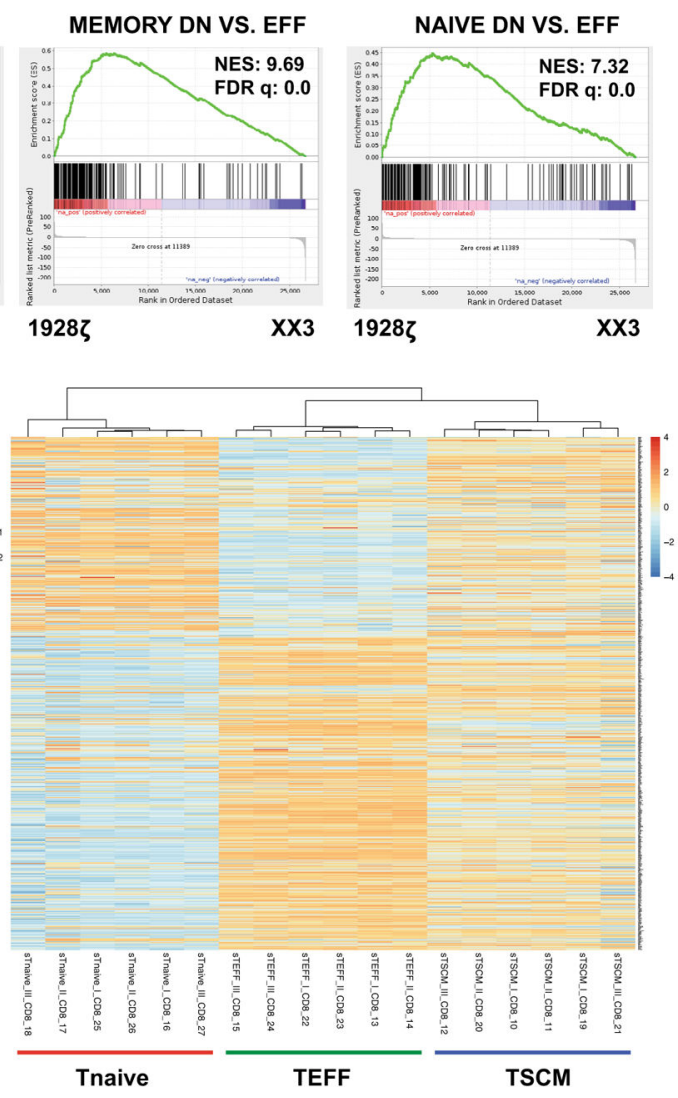

Extended Data Fig. 8 I. Transcriptional profiles of TRAC-encoded $1928 \zeta$ mutants and sorted control $\mathrm{T}$ cells.

a, Principal component analysis (PCA) of global transcriptional profiles of $\mathrm{CD} 8^{+}$ TRAC-1XX, TRAC-XX3 and TRAC-1928C after stimulation with CD19 target cells (left) and of sorted control $\mathrm{T}$ cell subsets (right): $\mathrm{T}_{\mathrm{N}}, \mathrm{T}_{\mathrm{SCM}}$ and $\mathrm{T}_{\mathrm{EFF}}$. Experiment was performed in technical triplicates for each CAR construct and in six replicates for each control subset. b, Representative GSEA enrichment plot (GSE10239), demonstrating downregulation of memory- relative to effector-related genes and naive- relative to effector-related genes in $1928 \zeta$ versus $1 X X$ and in $1928 \zeta$ versus XX3 ( $n=3$ mice per group). c, Heat map of 900 
differentially expressed genes among $\mathrm{CD}^{+} \mathrm{T}$ cell subsets as described by Gattinoni et $\mathrm{al}^{21}$ (left) compared to differential gene expression of sorted control $\mathrm{T}$ cell subsets $\left(\mathrm{T}_{\mathrm{EFF}}, \mathrm{T}_{\mathrm{SCM}}\right.$ and $\left.\mathrm{T}_{\mathrm{N}}\right)^{21}$. 
a

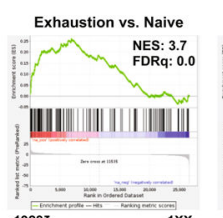

19285

Exhaustion vs. Naive
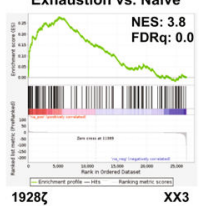

Exhaustion vs. Naive

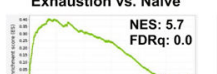

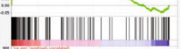

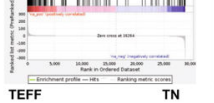

C

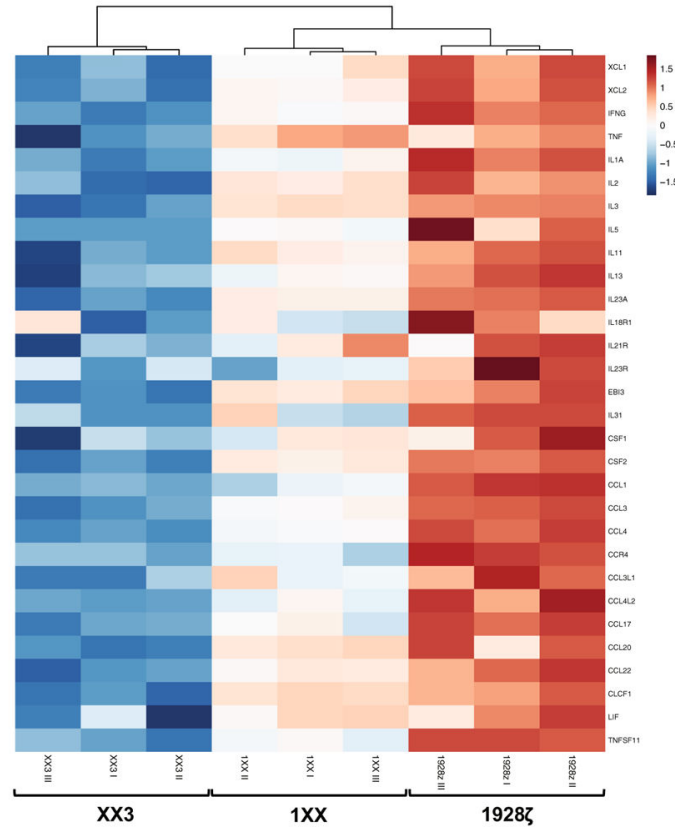

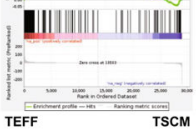

b

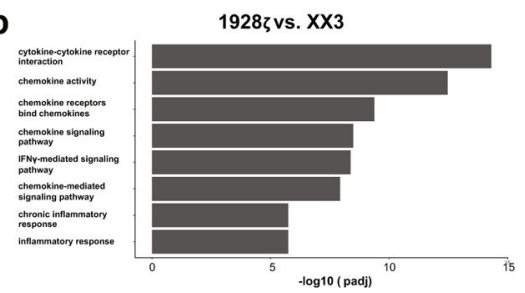

Exhaustion vs. Memory
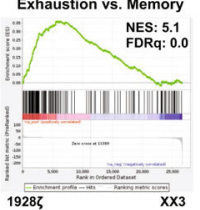

Exhaustion vs. Memory

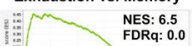

때||||| || || ||
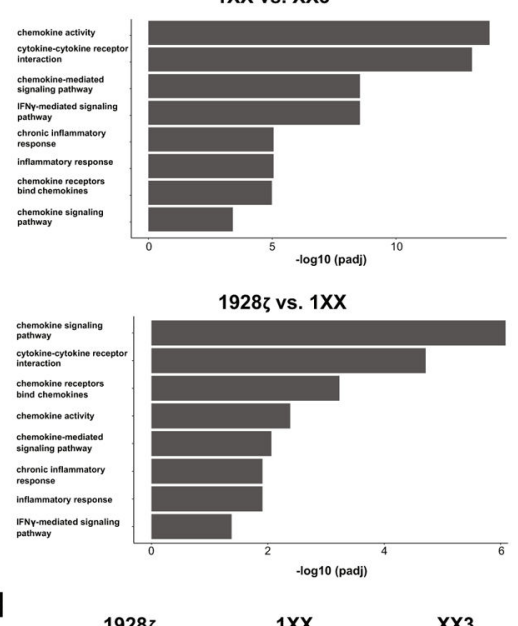

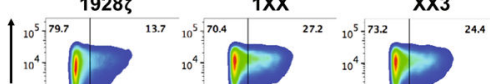

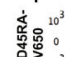
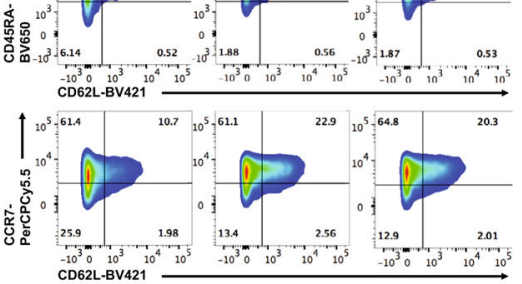

1

\begin{tabular}{l|l|l|l|l|l}
\hline 6.23 & 28.8 & $10^{5} \cdot 2.45$ & 20.4 & $10^{5} \cdot 2.47$ & 20.2
\end{tabular}

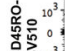

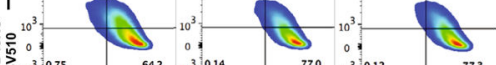

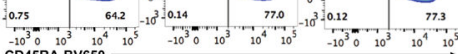

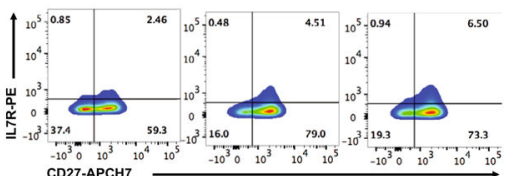

Extended Data Fig. 9 I. Impact of CD3 $\zeta$ ITAM mutations in TRAC-1928 $\zeta$ on T cell differentiation state and effector profile.

a, GSEA of a signature of the top 200 genes upregulated in exhausted CD8 T cells relative to naive or memory CD8 T cells as derived from GSE41867, demonstrating enrichment of exhaustion signature in $T R A C-1928 \zeta$ versus $T R A C-1 \mathrm{XX}$ or $T R A C-\mathrm{XX} 3$ and in the sorted control $\mathrm{T}_{\mathrm{EFF}}$ versus $\mathrm{T}_{\mathrm{N}}$ and $\mathrm{T}_{\mathrm{SCM}}$. Experiment was performed in technical triplicates for each CAR construct and in six replicates for each control subset. b, Gene ontology analysis demonstrating significantly enriched gene sets associated with inflammation, cytokine and chemokine signaling in $1928 \zeta$ versus XX3, 1 XX versus XX3 and $1928 \zeta$ versus 1 XX $(n=$ 
3). Transcriptional analysis was performed after CAR gene integration into the TRAClocus of naive T cells and stimulation with $\mathrm{CD} 19^{+}$target cells. Results are shown in order of significance as $-\log _{10}$ (corrected $P$ value). $P$ values were determined by a one-tailed Fisher's exact test and the Benjamini-Hochberg method was used to correct for multiple hypotheses testing. c, Heat map of selected differentially expressed genes between CAR constructs related to inflammation, cytokine and chemokine activity. d, Flow cytometric analysis of T cell differentiation state on CD8 ${ }^{+}$CAR T cells after stimulation with CD19 antigen (representative for $n=2$ independent experiments with similar results). 
a
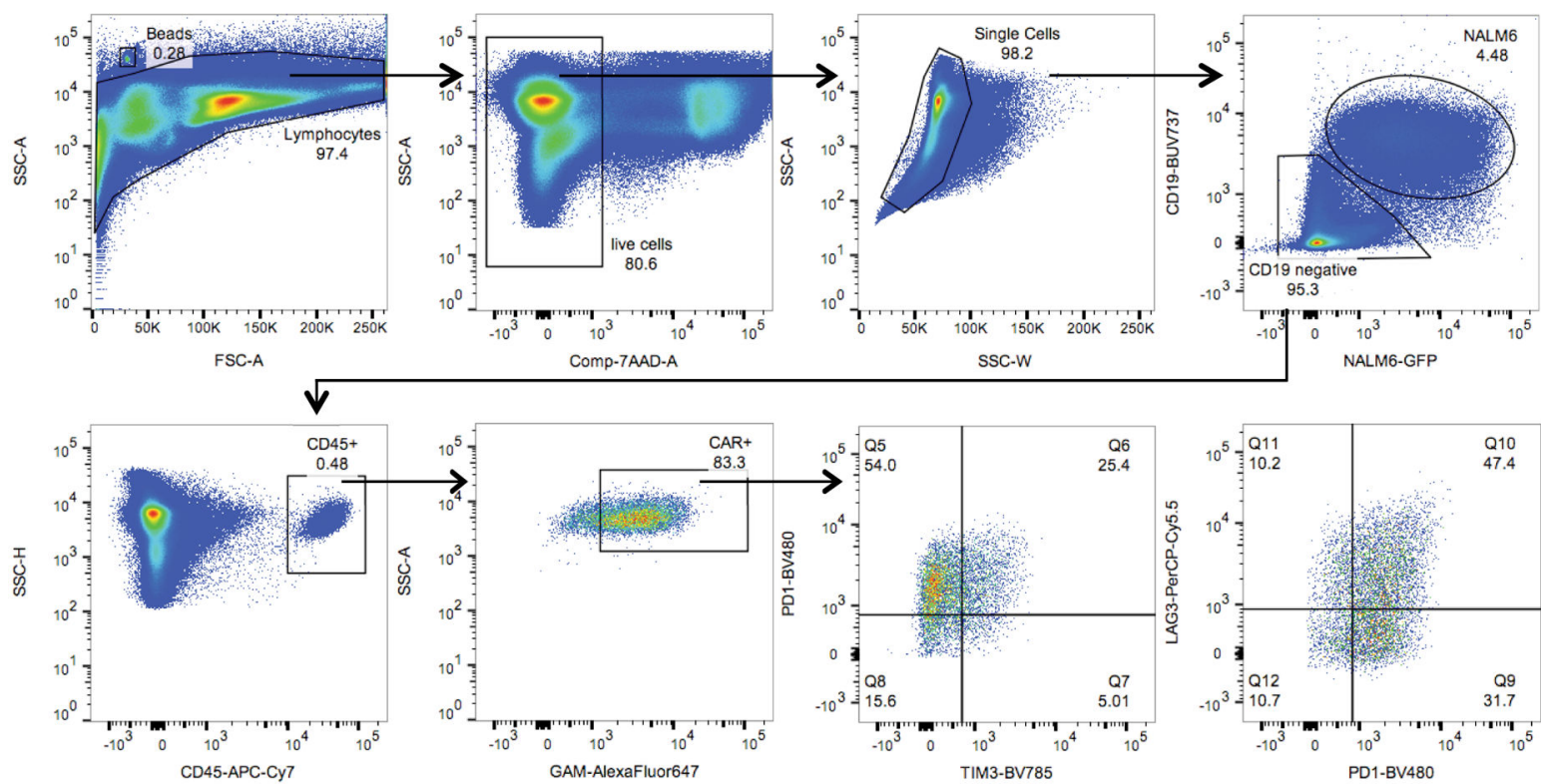

b
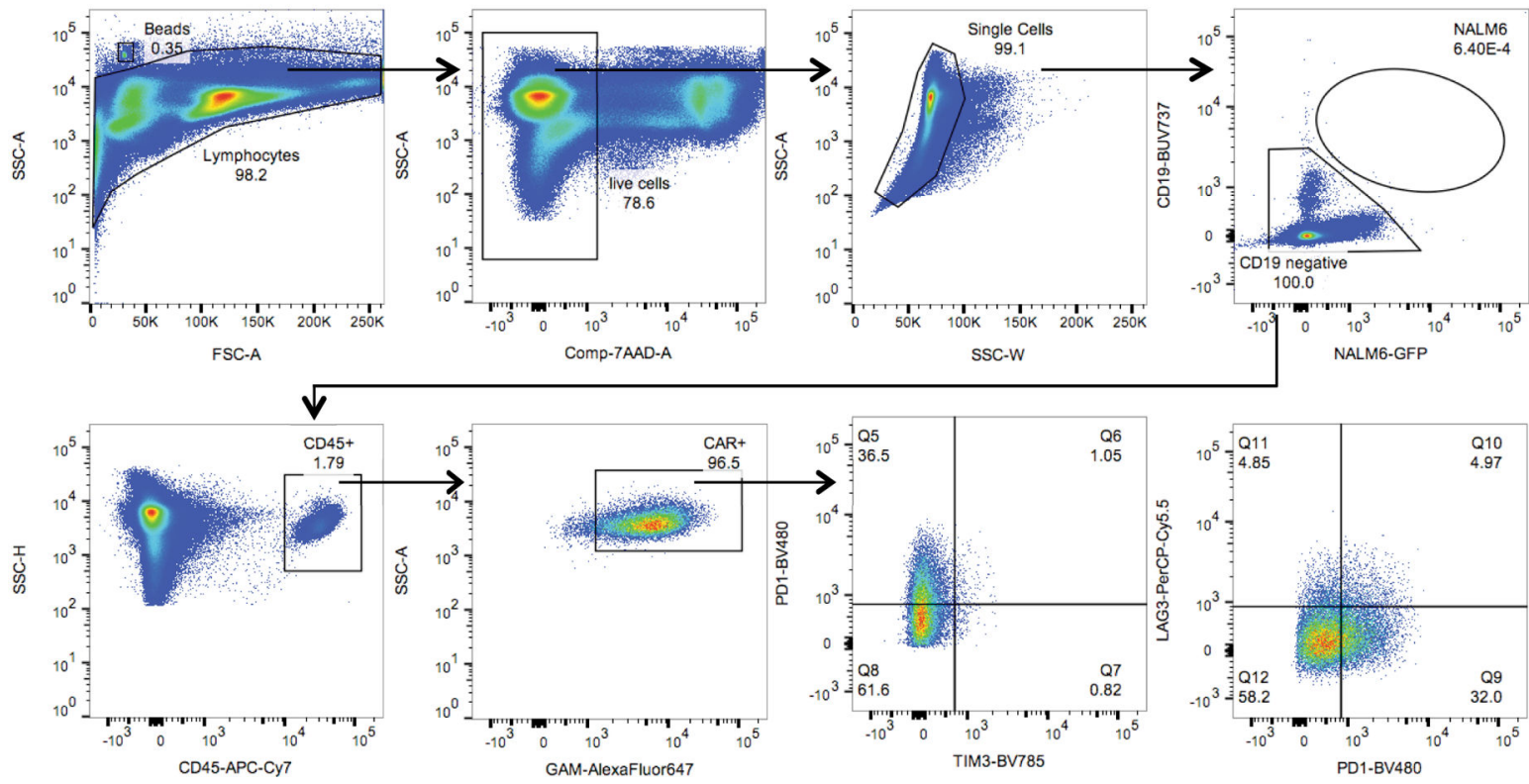

Extended Data Fig. 10 I. Gating strategy to analyze CAR T cells obtained from bone marrow of treated mice.

a,b Representative flow cytometric analysis of $T R A C-1928 \zeta$ (a) compared to $T R A C$-1XX

(b) on day 17 post CAR infusion. Placement of gating was based on FMO controls.

\section{Acknowledgements}

We thank J. Mansilla-Soto, S. van der Stegen, F. Perna, and other Sadelain laboratory members (Memorial Sloan Kettering Cancer Center) for helpful and critical discussions and G. Gunset for excellent technical assistance. We thank the following Memorial Sloan Kettering Cancer Center (MSKCC) core facilities for the excellent support: SKI Flow Cytometry core facility; animal facility; bioinformatics core; and integrated genomics operation core, 
funded by the NCI Cancer Center Support Grant (P30 CA08748), Cycle for Survival, and the Marie-Josée and Henry R. Kravis Center for Molecular Oncology. We thank the Care-for-Rare Foundation (J.F.), the German Research Foundation (DFG) (J.F.), the Edythe Griffinger Fellowship (J.S.), and the Louis V. Gerstner Jr. Graduate School of Biomedical Sciences (A.D.) for their support. This work was in part supported by the Lake Road Foundation, the Mr. William H. and Mrs. Alice Goodwin and the Commonwealth Foundation for Cancer Research, the Lymphoma and Leukemia Society, and the MSKCC Support Grant/Core Grant (P30 CA008748).

\section{References}

1. Sadelain M, Rivière I \& Riddell S Therapeutic T cell engineering. Nature 545, 423-431 (2017). [PubMed: 28541315]

2. Maher J, Brentjens RJ, Gunset G, Rivière I \& Sadelain M Human T-lymphocyte cytotoxicity and proliferation directed by a single chimeric TCRzeta/CD28 receptor. Nat. Biotechnol. 20, 70-75 (2002). [PubMed: 11753365]

3. Brentjens RJ et al. CD19-targeted T cells rapidly induce molecular remissions in adults with chemotherapy-refractory acute lymphoblastic leukemia. Sci. Transl. Med. 5, 177ra38 (2013).

4. Lee DW et al. T cells expressing CD19 chimeric antigen receptors for acute lymphoblastic leukaemia in children and young adults: a phase 1 dose-escalation trial. Lancet 385, 517-528 (2015). [PubMed: 25319501]

5. Park JH et al. Long-term follow-up of CD19 CAR therapy in acute lymphoblastic leukemia. N. Engl. J. Med. 378, 449-459 (2018). [PubMed: 29385376]

6. Neelapu SS et al. Axicabtagene Ciloleucel CAR T-cell therapy in refractory large B-cell lymphoma. N. Engl. J. Med. 377, 2531-2544 (2017). [PubMed: 29226797]

7. Sadelain M CD19 CAR T cells. Cell 171, 1471 (2017). [PubMed: 29245005]

8. Zhao $\mathrm{Z}$ et al. Structural design of engineered costimulation determines tumor rejection kinetics and persistence of CAR T cells. Cancer Cell 28, 415-428 (2015). [PubMed: 26461090]

9. Youngblood B, Davis CW \& Ahmed R Making memories that last a lifetime: heritable functions of self-renewing memory CD8 T cells. Int. Immunol. 22, 797-803 (2010). [PubMed: 20732857]

10. Wherry EJ \& Kurachi M Molecular and cellular insights into T cell exhaustion. Nat. Rev. Immunol. 15, 486-499 (2015). [PubMed: 26205583]

11. Acuto O \& Michel F CD28-mediated co-stimulation: a quantitative support for TCR signalling. Nat. Rev. Immunol. 3, 939-951 (2003). [PubMed: 14647476]

12. Smith-Garvin JE, Koretzky GA \& Jordan MS T cell activation. Annu. Rev. Immunol. 27, 591-619 (2009). [PubMed: 19132916]

13. Love PE \& Hayes SM ITAM-mediated signaling by the T-cell antigen receptor. Cold Spring Harb. Perspect. Biol. 2, a002485 (2010). [PubMed: 20516133]

14. Kersh EN, Shaw AS \& Allen PM Fidelity of T cell activation through multistep T cell receptor zeta phosphorylation. Science 281, 572-575 (1998). [PubMed: 9677202]

15. Isakov $\mathrm{N}$ et al. ZAP-70 binding specificity to $\mathrm{T}$ cell receptor tyrosine-based activation motifs: the tandem SH2 domains of ZAP-70 bind distinct tyrosine-based activation motifs with varying affinity. J. Exp. Med. 181, 375-380 (1995). [PubMed: 7528772]

16. van Oers NS et al. The 21- and 23-kD forms of TCR zeta are generated by specific ITAM phosphorylations. Nat. Immunol. 1, 322-328 (2000). [PubMed: 11017104]

17. Chae WJ et al. Qualitatively differential regulation of $\mathrm{T}$ cell activation and apoptosis by $\mathrm{T}$ cell receptor zeta chain ITAMs and their tyrosine residues. Int. Immunol. 16, 1225-1236 (2004). [PubMed: 15302845]

18. Mukhopadhyay H, Cordoba SP, Maini PK, van der Merwe PA \& Dushek O Systems model of T cell receptor proximal signaling reveals emergent ultrasensitivity. PLoS Comput. Biol. 9, e1003004 (2013). [PubMed: 23555234]

19. Eyquem $J$ et al. Targeting a CAR to the TRAC locus with CRISPR/Cas9 enhances tumour rejection. Nature 543, 113-117 (2017). [PubMed: 28225754]

20. Brentjens RJ et al. Eradication of systemic B-cell tumors by genetically targeted human T lymphocytes co-stimulated by CD80 and interleukin-15. Nat. Med. 9, 279-286 (2003). [PubMed: 12579196] 
21. Gattinoni L et al. A human memory T cell subset with stem cell-like properties. Nat. Med. 17, 1290-1297 (2011). [PubMed: 21926977]

22. Chang JT, Wherry EJ \& Goldrath AW Molecular regulation of effector and memory T cell differentiation. Nat. Immunol. 15, 1104-1115 (2014). [PubMed: 25396352]

23. Yu B et al. Epigenetic landscapes reveal transcription factors that regulate $\mathrm{CD}^{+} \mathrm{T}$ cell differentiation. Nat. Immunol. 18, 573-582 (2017). [PubMed: 28288100]

24. Kaech SM \& Cui W Transcriptional control of effector and memory $\mathrm{CD}^{+} \mathrm{T}$ cell differentiation. Nat. Rev. Immunol. 12, 749-761 (2012). [PubMed: 23080391]

25. Ichii $\mathrm{H}$ et al. Role for Bcl-6 in the generation and maintenance of memory $\mathrm{CD} 8^{+} \mathrm{T}$ cells. Nat. Immunol. 3, 558-563 (2002). [PubMed: 12021781]

26. Zhou X \& Xue HH Cutting edge: generation of memory precursors and functional memory CD $8^{+}$ $\mathrm{T}$ cells depends on $\mathrm{T}$ cell factor- 1 and lymphoid enhancer-binding factor-1. J. Immunol. 189, 2722-2726 (2012). [PubMed: 22875805]

27. Carlson CM et al. Kruppel-like factor 2 regulates thymocyte and T-cell migration. Nature 442 , 299-302 (2006). [PubMed: 16855590]

28. Bai A, Hu H, Yeung M \& Chen J Kruppel-like factor 2 controls T cell trafficking by activating Lselectin (CD62L) and sphingosine-1-phosphate receptor 1 transcription. J. Immunol. 178, 76327639 (2007). [PubMed: 17548599]

29. Daniels MA \& Teixeiro E TCR signaling in T cell memory. Front. Immunol. 6, 617 (2015). [PubMed: 26697013]

30. Fraietta JA et al. Determinants of response and resistance to CD19 chimeric antigen receptor (CAR) T cell therapy of chronic lymphocytic leukemia. Nat. Med. 24, 563-571 (2018). [PubMed: 29713085]

31. Sommermeyer D et al. Chimeric antigen receptor-modified T cells derived from defined $\mathrm{CD}^{+}{ }^{+}$and $\mathrm{CD}^{+}{ }^{+}$subsets confer superior antitumor reactivity in vivo. Leukemia 30, 492-500 (2016). [PubMed: 26369987]

32. Zhao $\mathrm{Y}$ et al. A herceptin-based chimeric antigen receptor with modified signaling domains leads to enhanced survival of transduced T lymphocytes and antitumor activity. J. Immunol. 183, 55635574 (2009). [PubMed: 19843940]

33. James JR Tuning ITAM multiplicity on T cell receptors can control potency and selectivity to ligand density. Sci. Signal. 11, eaan1088 (2018). [PubMed: 29789296]

34. Kochenderfer JN, Yu Z, Frasheri D, Restifo NP \& Rosenberg SA Adoptive transfer of syngeneic T cells transduced with a chimeric antigen receptor that recognizes murine CD19 can eradicate lymphoma and normal B cells. Blood 116, 3875-3886 (2010). [PubMed: 20631379]

35. Rivière I, Brose K \& Mulligan RC Effects of retroviral vector design on expression of human adenosine deaminase in murine bone marrow transplant recipients engrafted with genetically modified cells. Proc. Natl Acad. Sci. USA 92, 6733-6737 (1995). [PubMed: 7624312]

36. Gong MC et al. Cancer patient $\mathrm{T}$ cells genetically targeted to prostate-specific membrane antigen specifically lyse prostate cancer cells and release cytokines in response to prostate-specific membrane antigen. Neoplasia 1, 123-127 (1999). [PubMed: 10933046]

37. Brentjens RJ et al. Genetically targeted T cells eradicate systemic acute lymphoblastic leukemia xenografts. Clin. Cancer Res. 13, 5426-5435 (2007). [PubMed: 17855649]

38. Ghosh A et al. Adoptively transferred TRAIL ${ }^{+} \mathrm{T}$ cells suppress GVHD and augment antitumor activity. J. Clin. Invest. 123, 2654-2662 (2013). [PubMed: 23676461]

39. Gade TP et al. Targeted elimination of prostate cancer by genetically directed human $\mathrm{T}$ lymphocytes. Cancer Res. 65, 9080-9088 (2005). [PubMed: 16204083]

40. Dobin A et al. STAR: ultrafast universal RNA-seq aligner. Bioinformatics 29, 15-21 (2013). [PubMed: 23104886] 

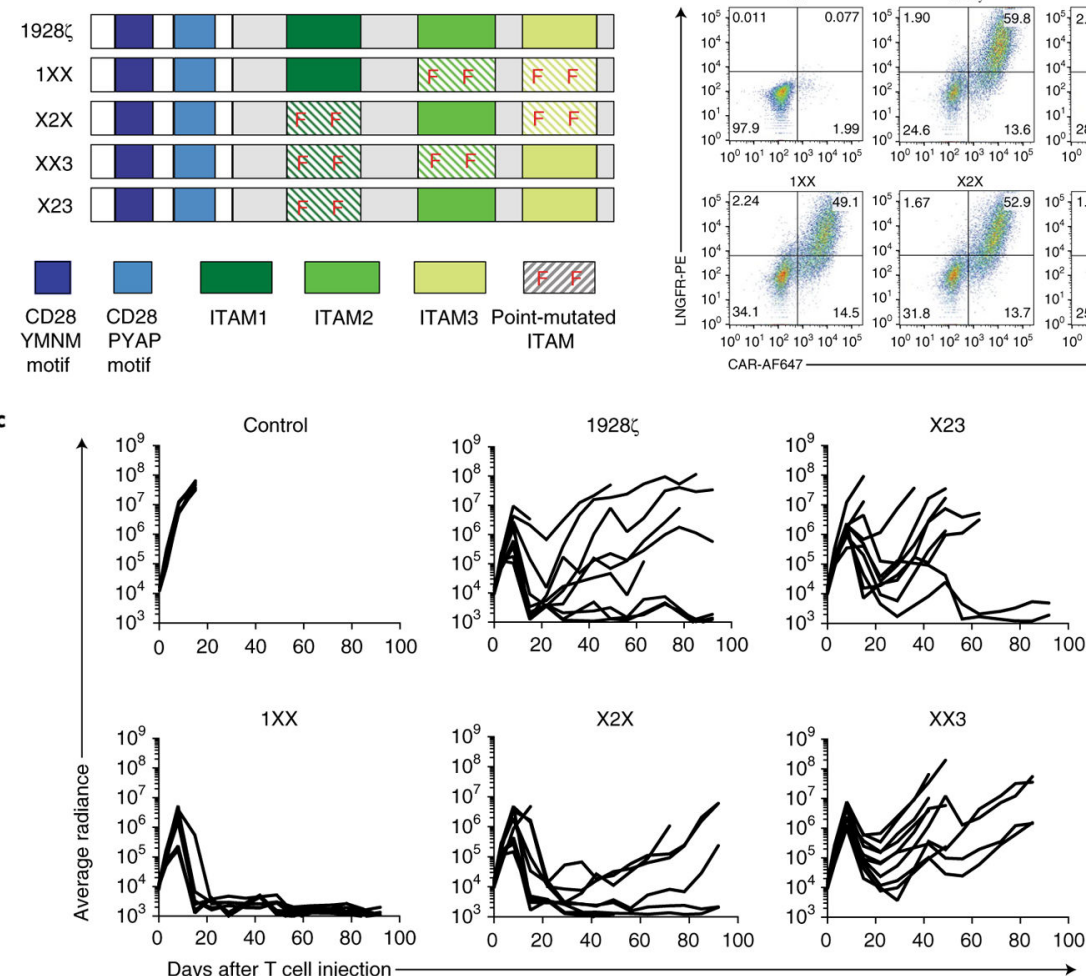
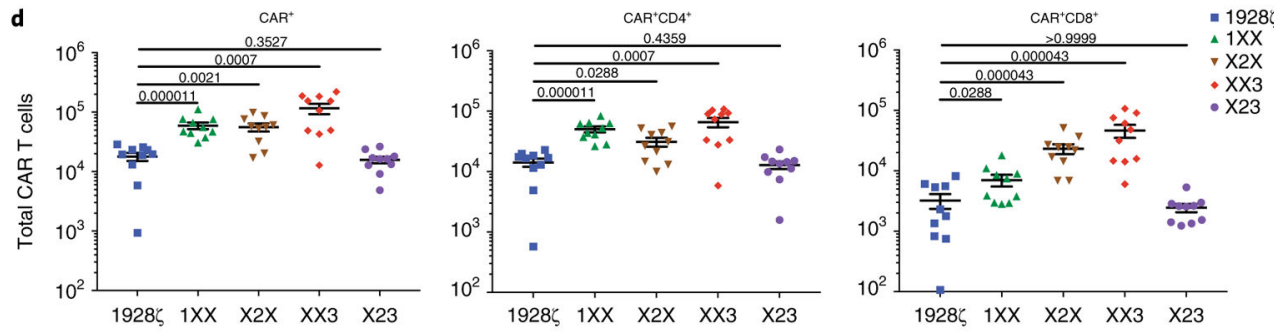

$\mathrm{CAR}^{+} \mathrm{CD} 4^{+} \mathrm{CD} 62 \mathrm{~L}^{+} \mathrm{CD} 45 \mathrm{RA}^{-}$

$\mathrm{CAR}^{+} \mathrm{CD} 4^{+} \mathrm{CD} 2 \mathrm{~L}^{-} \mathrm{CD} 45 \mathrm{RA}^{+}$

$\mathrm{CAR}^{+} \mathrm{CD} 8^{+} \mathrm{CD} 62 \mathrm{~L}^{+} \mathrm{CD} 45 \mathrm{RA}^{-}$
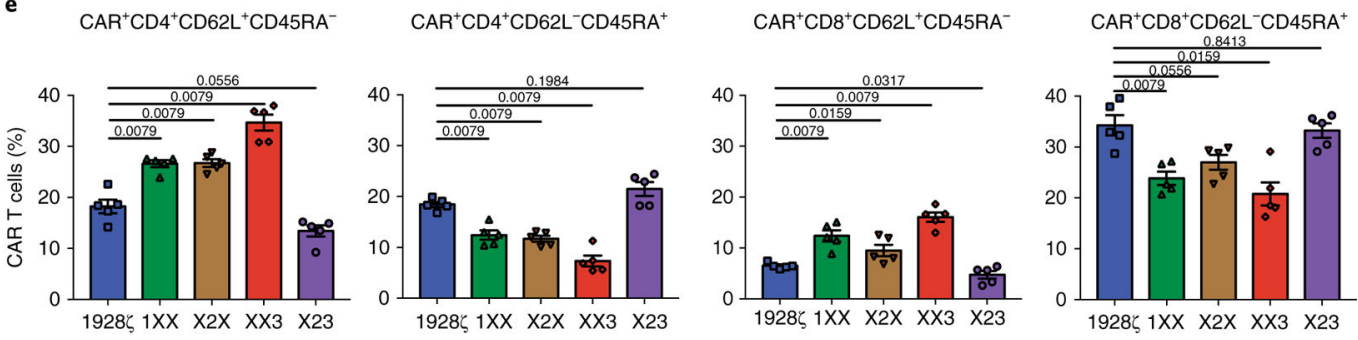

Fig. 1 I. 1928 $\zeta$ ITAMs differentially regulate CAR $T$ cell potency.

a, Cytoplasmic regions of wild-type and mutated $1928 \zeta$ CARs. The $\zeta$ chain of the $1928 \zeta$ CAR was mutated in one or two of its three $\zeta$ signaling domains, named ITAM1, ITAM2, and ITAM3, from a membrane-proximal to a membrane-distal direction. In 1XX, X2X, $\mathrm{XX} 3$, and X23 CARs, the two tyrosines (Y) in the respective ITAM are point-mutated to two phenylalanines (F) for the indicated ITAMs. b, Flow cytometric analysis showing expression levels of CAR and LNGFR for the constructs depicted in a. Data are representative of at least five independent experiments with similar results. Untransduced T (UT) cells were used as the control. c-e, Nalm6-bearing mice were treated with $5 \times 10^{4} \mathrm{CAR}^{+} \mathrm{T}$ cells. $\mathbf{c}$, 
Tumor burden (average radiance) of mice is shown, comparing the in vivo efficacy of wildtype 1928 $, 1 \mathrm{XX}, \mathrm{X} 2 \mathrm{X}, \mathrm{XX} 3$, and X23 ( $n=10$ mice per group, results were pooled from two independent experiments). Control refers to untreated mice $(n=6)$. d, Number of CAR $\mathrm{T}$ cells in the bone marrow of mice $17 \mathrm{~d}$ post-infusion (results were pooled from two independent experiments, $n=10$ mice per group). e, Phenotype of CAR T cells in the bone marrow of mice $10 \mathrm{~d}$ after CAR infusion, as demonstrated by the percentage of $\mathrm{T}_{\mathrm{CM}}$ and $\mathrm{T}_{\mathrm{EFF}}$ cells. Representative results of two independent experiments are shown $(n=5$ mice per group). All data are mean \pm s.e.m. In $\mathbf{d}$ and $\mathbf{e}, P$ values were determined by two-tailed Mann-Whitney $U$-tests. See also Extended Data Figs. 1 and 2. 


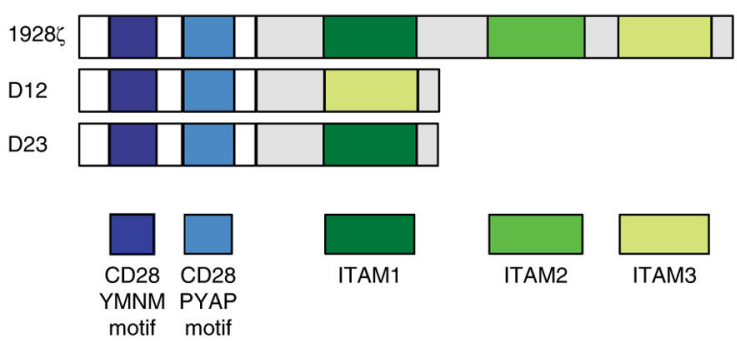

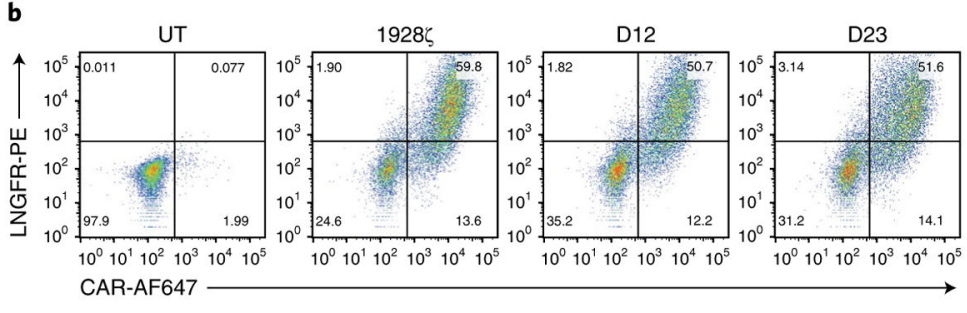
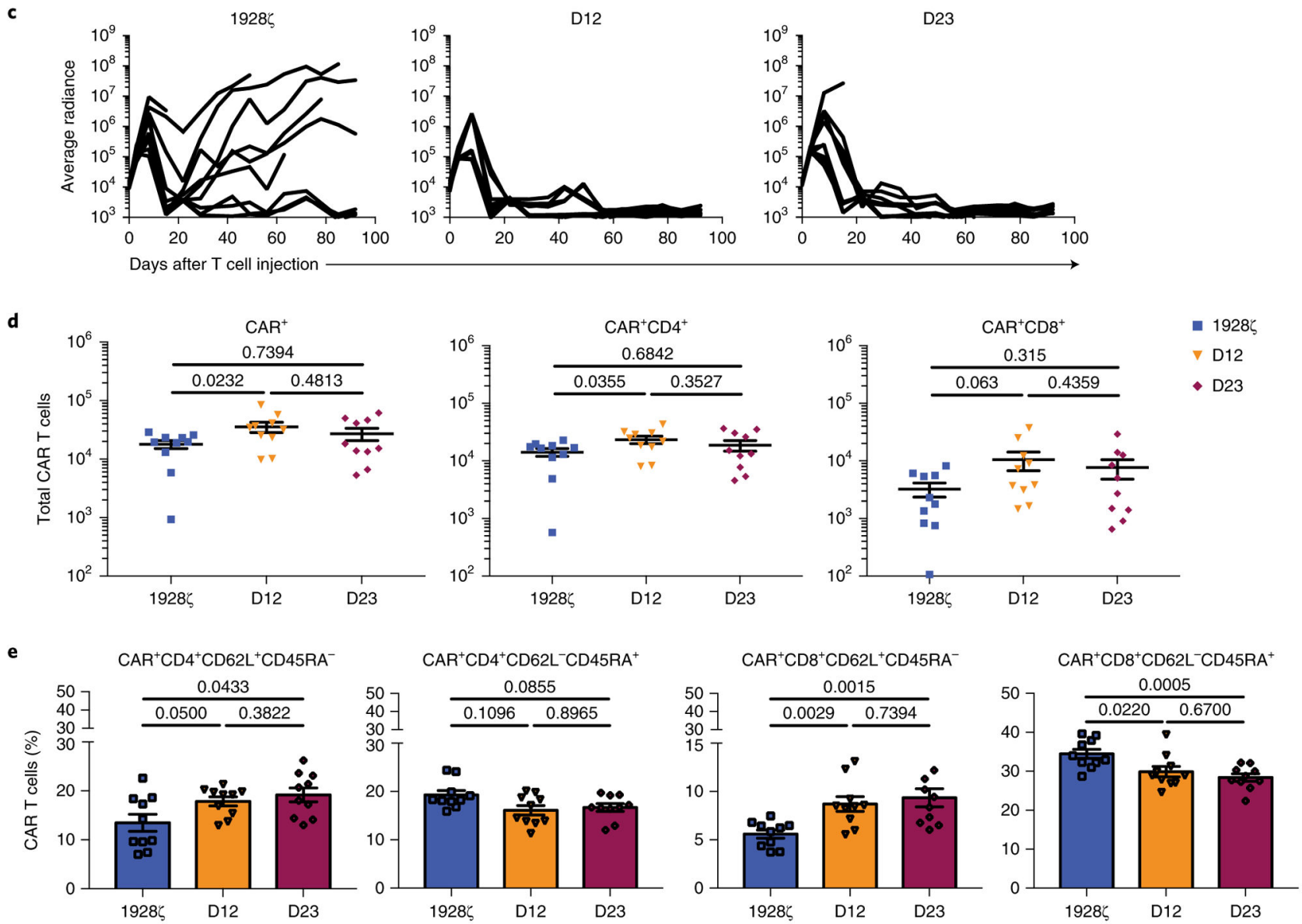

Fig. 2 I. ITAM position within 1928 $\zeta$ CARs determines antitumor efficacy.

a, Cytoplasmic domains of $1928 \zeta$ CARs with deletions in the CD3 $\zeta$ chain. In D12, deletion mutations remove ITAM1 and ITAM2, while ITAM2 and ITAM3 are removed in D23. b,

Flow cytometric analysis showing the expression levels of CAR and LNGFR for the indicated constructs. Data are representative of at least five independent experiments with similar results. UT, untransduced T cells were used as the control. c-e, Nalm6-bearing mice were treated with $5 \times 10^{4} \mathrm{CAR}^{+} \mathrm{T}$ cells. c, Tumor burden (average radiance) of mice treated

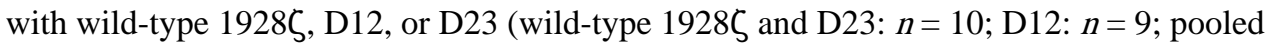
data from two independent experiments). d, Number of CAR T cells in the bone marrow of mice $17 \mathrm{~d}$ post-infusion (results were pooled from two independent experiments, $n=10$ mice per group). e, Phenotype of CAR T cells in the bone marrow of mice $10 \mathrm{~d}$ after CAR 
infusion, as demonstrated by the percentage of $\mathrm{T}_{\mathrm{CM}}$ and $\mathrm{T}_{\mathrm{EFF}}$ cells (pooled data from two independent experiments, $n=10$ mice per group). All data are mean \pm s.e.m. In $\mathbf{d}$ and $\mathbf{e}, P$ values were determined by two-tailed Mann-Whitney $U$-tests. See also Extended Data Figs. 3 and 4. 
a

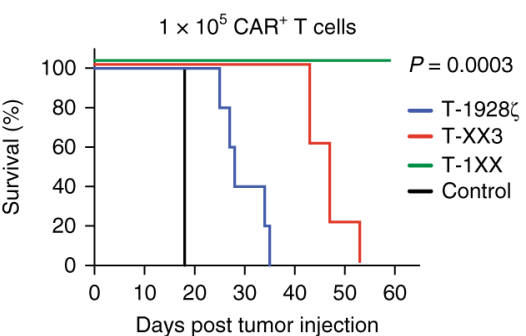

c

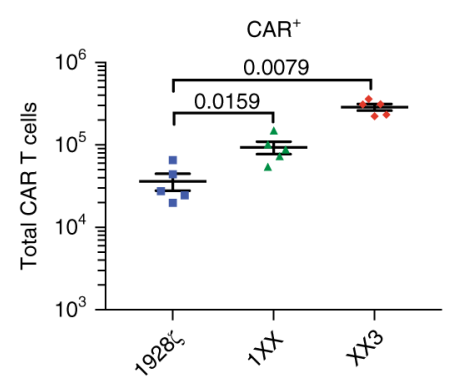

e

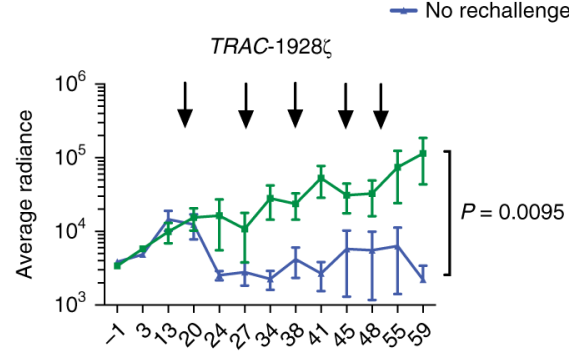

Days after $T$ cell injection b

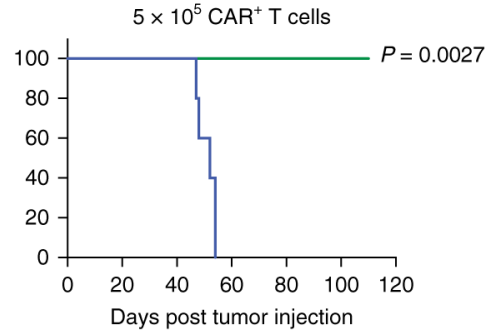

d
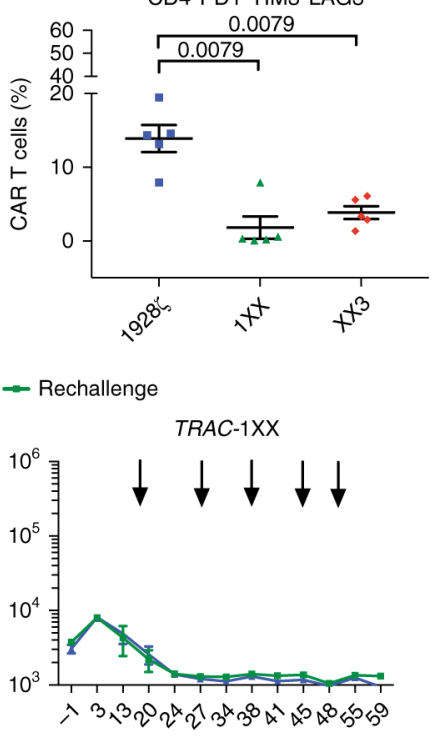

$\mathbf{g}$

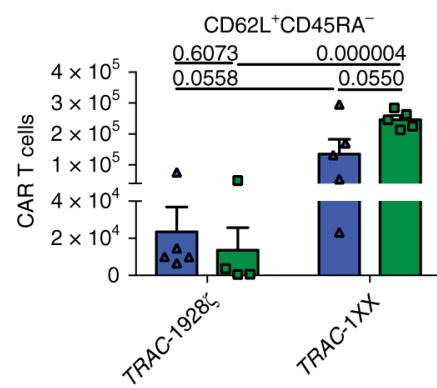

$\Delta$ No rechallenge $\square$ Rechallenge

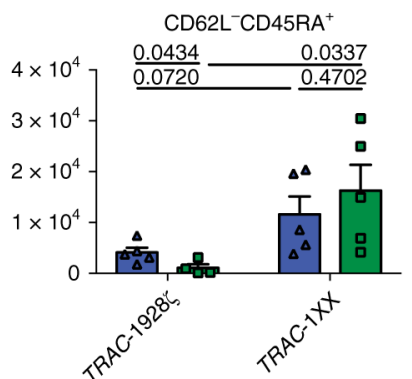

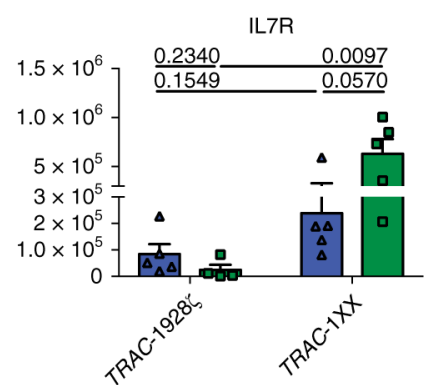
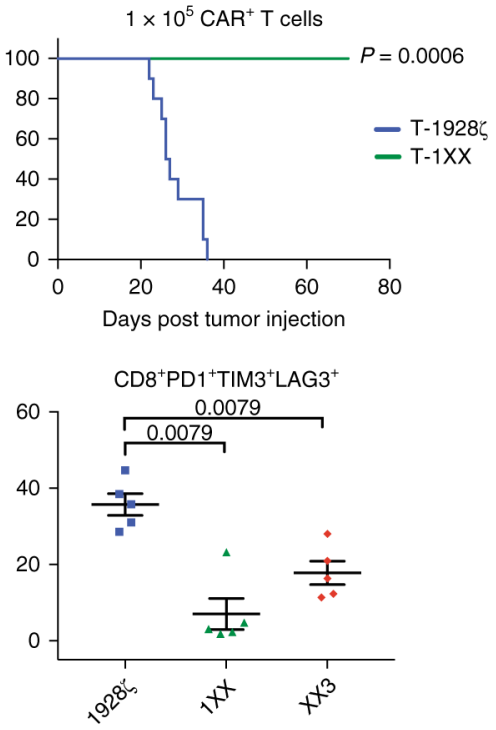

f

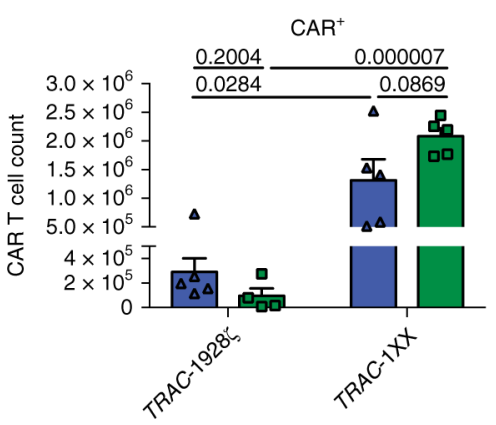

Fig. 3 I. TRAC-1XX augments $T$ cell potency by decreasing $T$ cell exhaustion and developing into long-lived memory $\mathbf{T}$ cells with effective recall responses.

a-d, Nalm6-bearing mice were treated with $1 \times 10^{5}$ or $5 \times 10^{5}$ TRAC-CAR T cells. a, Kaplan-Meier analysis of survival of mice treated with $1 \times 10^{5} T R A C-1 \mathrm{XX}$ or TRAC-XX3

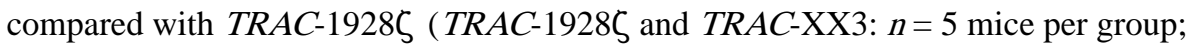

TRAC-1XX: $n=7)$. Control refers to untreated mice $(n=3)$. $P$ values were determined by a one-sided log-rank Mantel-Cox test. b, Kaplan-Meier analysis of survival of mice treated

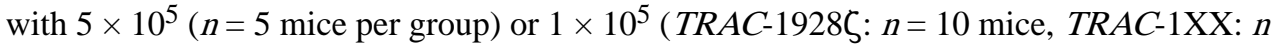
$=5$ mice) $T R A C$-CAR T cells. $P$ values were determined by a one-sided log-rank MantelCox test. c,d, The number of cells (c) and expression of the exhaustion markers $\mathrm{PD}^{+}{ }^{+} \mathrm{TIM} 3^{+} \mathrm{LAG}^{+}$on bone marrow $\mathrm{CAR} \mathrm{T}$ cells $(\mathbf{d})$ were determined for $T R A C-1 \mathrm{XX}$ and 
TRAC-XX3 and compared with TRAC-1928C. Data are shown as mean \pm s.e.m., and each symbol denotes an individual mouse ( $n=5$ mice per group). $P$ values were determined by two-tailed Mann-Whitney $U$-tests. $\mathbf{e}-\mathbf{h}$, Nalm6-bearing mice were treated with $5 \times 10^{5}$ $T R A C$-edited naive T cells and were rechallenged with Nalm6 cells ( $n=5$ mice per group), as indicated by the arrows. No further rechallenge with tumor was performed for the

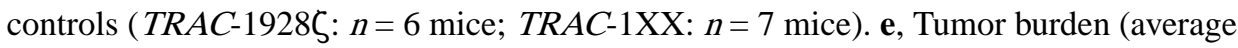
radiance) of mice, comparing the in vivo efficacy of TRAC-1928 5 and TRAC-1XX following tumor rechallenge versus no further rechallenge. All data are mean \pm s.e.m. A two-tailed unpaired Student's $t$-test was used for the statistical analysis of tumor burden at day 59 post-CAR administration (TRAC-1928C: $n=4$; TRAC-1XX: $n=5$ ). f,g, The number of total CAR T cells (f), $\mathrm{T}_{\mathrm{CM}}, \mathrm{T}_{\mathrm{EFF}}$, and IL7R ${ }^{+}$CAR T cells $(\mathrm{g})$ in the bone marrow of treated mice $63 \mathrm{~d}$ post-CAR administration (rechallenge: TRAC-1928 $: n=4$ mice, TRAC-1XX: $n=5$ mice; no rechallenge: $n=5$ mice per group). All data are mean \pm s.e.m.; a two-tailed unpaired Student's $t$-test was used for the statistical analysis. $\mathbf{h}$, Expression of the exhaustion markers $\mathrm{PD}^{+}{ }^{+} \mathrm{TIM}^{+}{ }^{+} \mathrm{LAG}^{+}$on bone marrow CAR T cells. All data are mean \pm s.e.m. $P$ values were determined by a two-tailed unpaired Student's $t$-test. See also Extended Data Figs. 5, 6, and 7. 

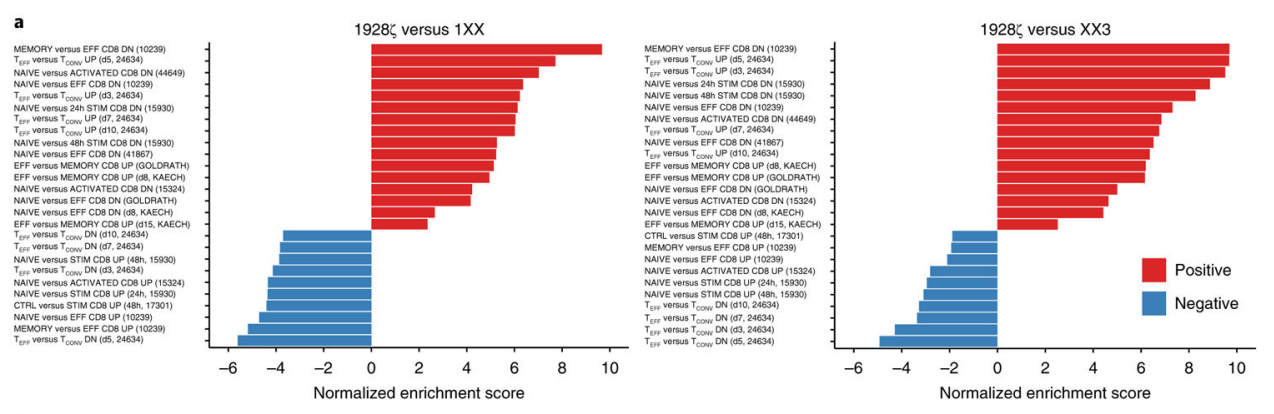

b
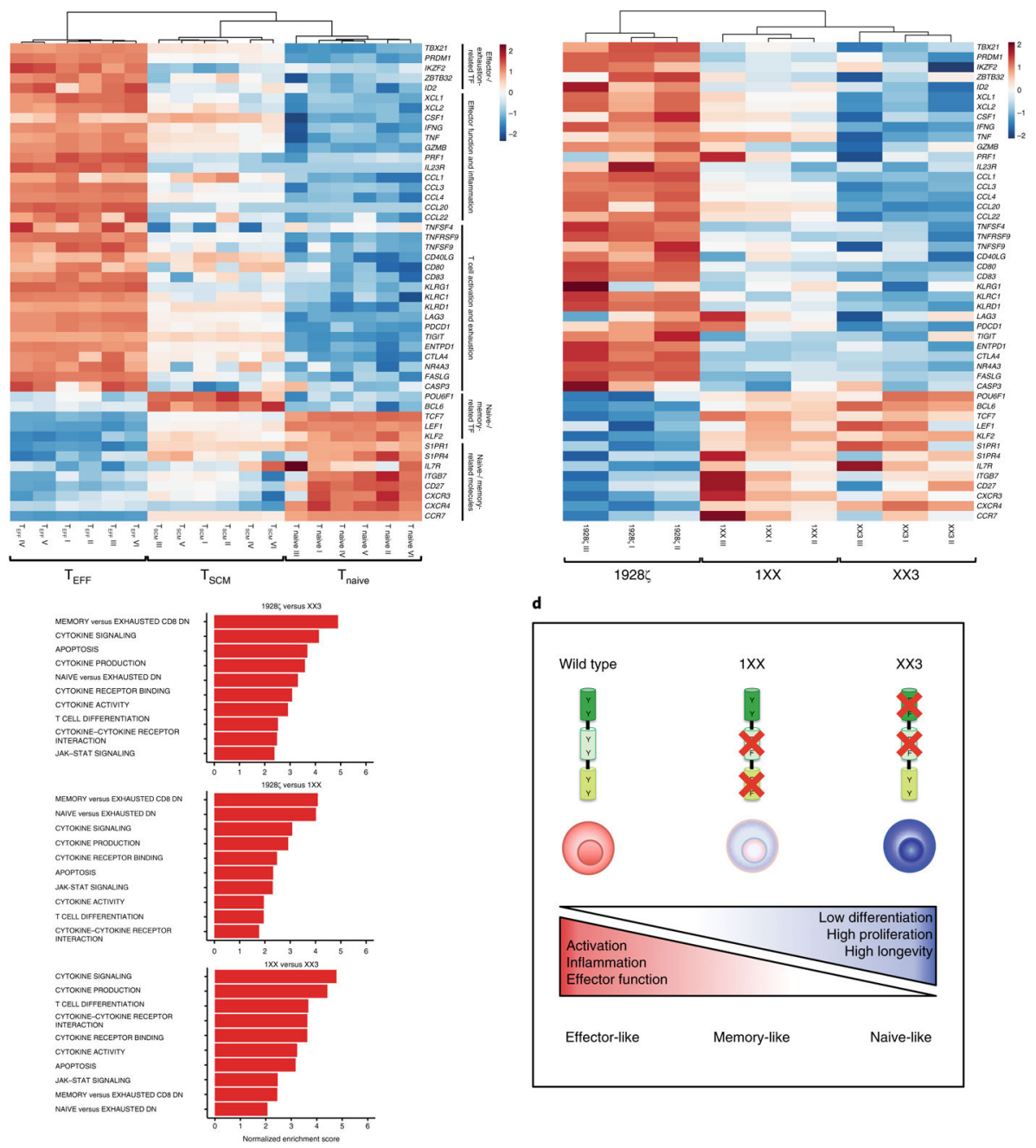

Fig. 4 I. CD3 $\zeta$ ITAM mutations in 1928 $\zeta$ CARs establish distinct transcriptional signatures. Gene expression profiles of $\mathrm{CD}^{+} T R A C-1928 \zeta, T R A C-1 \mathrm{XX}$, and TRAC-XX3 CAR T cells (initially sorted naive T cells) 24 h post stimulation with $\mathrm{CD} 19^{+}$target cells. a, Normalized enrichment score of significantly up- or downregulated gene sets in $1928 \zeta$ versus $1 \mathrm{XX}$ and $1928 \zeta$ versus XX3 ( $n=3$ replicates per group) as determined by GSEA using the MSigDB C7 gene ontology sets. For all pathways, the false discovery rate (FDR) $q \leq 0.02$. GSE datasets are indicated in parentheses. stim, stimulated. b, Differentially expressed genes (FDR $q<0.05)$ between sorted effector and naive/memory T cells (left) ( $n=6$ replicates per group) and heat map demonstrating the expression profiles of the same genes for CAR T 
cells ( $n=3$ replicates per group). TF, transcription factor. $\mathbf{c}$, Normalized enrichment score of significantly enriched gene sets (FDR $q \leq 0.03$ ) related to phenotypic and functional T cell

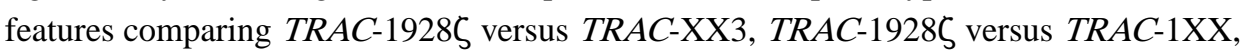
and $T R A C$ - $1 \mathrm{XX}$ versus $T R A C$-XX 3 as identified with GSEA analysis ( $n=3$ replicates per group). JAK-STAT, Janus kinase-signal transducer and activator of transcription. d, Impact of defined CD3 $\zeta$ ITAM mutations in $1928 \zeta$ CAR T cells on effector- and memoryassociated $\mathrm{T}$ cell attributes. 1XX CAR T cells display balanced effector and memory traits. See also Extended Data Figs. 8 and 9. 